\title{
Macromolecular tissue volume mapping of lateral geniculate nucleus subdivisions in living human brains
}

\section{Author Information:}

Hiroki Oishi $^{\mathrm{a}, \mathrm{b})^{\star}}$, Hiromasa Takemura ${ }^{\mathrm{a}, \mathrm{b})}$, Kaoru Amano ${ }^{\mathrm{a}, \mathrm{b})}$

\section{Affiliation:}

a) Center for Information and Neural Networks (CiNet), National Institute of Information and Communications Technology, and Osaka University, Suita 565-0871, Japan

b) Graduate School of Frontier Biosciences, Osaka University, Suita 565-0871, Japan

* To whom correspondence should be addressed: elanvital0014@gmail.com

Author contributions: Conceptualization: HO HT KA, Methodology: HO HT, Software: $\mathrm{HO}$, Validation: $\mathrm{HO}$, Formal analysis: $\mathrm{HO}$, Investigation: $\mathrm{HO}$, Data curation: $\mathrm{HO}$, Writingoriginal draft: HO HT KA, Writing-review and editing: HO HT KA, Visualization: HO, Supervision: HT KA, Project administration: HT KA, Funding acquisition: HO HT KA. 


\section{Abstract}

The human lateral geniculate nucleus (LGN) is composed mainly of the magnocellular and parvocellular subdivisions. The non-invasive identification of these subdivisions is, however, difficult due to the small size of the LGN. Here we propose a method to identify these subdivisions by combining two structural MR measures: high-resolution protondensity weighted images and macromolecular tissue volume maps. We collected MRI data from 15 healthy subjects and found that the spatial organization of these subdivisions identified by our method was consistent with post-mortem histological data. Furthermore, the stimulus selectivity in these regions measured by functional MRI was consistent with physiological studies. These results suggest that macromolecular tissue volume mapping is a promising approach to evaluating the tissue properties of LGN subdivisions in living humans. This method potentially will enable neuroscientific and clinical hypotheses about the human LGN to be tested. 


\section{Introduction}

The lateral geniculate nucleus (LGN) plays an essential role in human visual processing by receiving visual inputs from retinal ganglion cells and transferring those signals to the primary visual cortex (Nassi and Callaway, 2009). Besides its function as a relay station, a growing body of evidence suggests that the LGN is involved in a wide range of visual functions, including eye-specific dominance and suppression during binocular rivalry (Haynes et al., 2005), visual attention (Ling et al., 2015; O'Connor et al., 2002; Schneider and Kastner, 2009), and visual perceptual learning (Yu et al., 2016). The LGN is also involved in the neuronal synchrony widely observed in the visual cortex (Hughes et al., 2004; Liu et al., 2012; Minami et al., 2020).

Human LGN is composed of six layers that are categorized into two major subdivisions, the magnocellular $(M)$ and parvocellular $(P)$ subdivisions. These subdivisions are clearly distinguishable on the basis of cell size (Hickey and Guillery, 1979; Gupta et al., 2006). A number of studies have suggested that the $M$ and $P$ subdivisions have complementary roles in visual processing by demonstrating distinct spatial, temporal, luminance, and chromatic stimuli preferences (Denison et al., 2014; Derrington and Lennie, 1984; Schiller et al., 1990; Usrey and Reid, 2000). According to psychophysical performances in response to specific types of visual stimuli and neural responses in the $M$ and $P$ subdivisions, these two subdivisions have been proposed to have distinct roles in attention (Yeshurun and Levy, 2003) and reading (Demb et al., 1998; Main et al., 2014; Stein, 2001). A psychophysical study using a motion coherence task also led to a hypothesis that the $M$ subdivision is damaged earlier than the $P$ subdivision as a consequence of glaucoma (Joffe et al., 1997). However, the hypotheses proposed by previous psychophysical studies remain speculative, because they are derived mostly from similarities between the neural responses of the $M$ and $P$ subdivisions and stimulidependent psychophysical performances without assessing the differences in functional and structural measurements between the two. Therefore, it is essential to establish noninvasive measurements to enable the direct comparison of neuroimaging data from the LGN subdivisions and psychophysical data, while also comparing the properties of the $M$ and $P$ subdivisions with those of other visual cortical areas.

Non-invasive neuroimaging-based measurements of the structural properties of human LGN subdivisions have not been established, because of the requirement for highresolution, quantitative MRI measurements for the LGN, which has a small volume around $180 \mathrm{~mm}^{3}$ (Mcketton et al., 2014). While functional MRI (fMRI) has been used to 
localize $\mathrm{M}$ and $\mathrm{P}$ subdivisions based upon the blood oxygenation level-dependent (BOLD) response selectivity for distinct visual stimuli (Denison et al., 2014; Zhang et al., $2016,2015)$, the spatial resolution $\left(1.25 \times 1.25 \times 1.2 \mathrm{~mm}^{3}-1.75 \times 1.75 \times 1.5 \mathrm{~mm}^{3}\right)$ and robustness of the measurements have been limited. fMRI measurements also require the use of visual stimuli, which limit the applicability for clinical populations with visual field loss.

To examine the properties of LGN subdivisions in individual living humans, here we propose a method by which the subdivisions can be identified using structural MRI measurements. To this end, we primarily used macromolecular tissue volume (MTV) mapping, which is a quantitative structural MRI method that is sensitive to a fraction of non-water macromolecules (Mezer et al., 2013). We hypothesized that the MTV fraction would enable the $M$ and $P$ subdivisions to be distinguished as (1) MTV is sensitive to lipids which constitute cell membranes and myelin (Filo et al., 2019; Mezer et al., 2013; Shtangel and Mezer, 2020) and (2) neuronal cell and myelin densities differ between the M and P subdivisions (Hassler, 1966; Pistorio et al., 2006; Yücel et al., 2003, 2000). The first step in our proposed method was to identify the location and contour of the whole LGN using high-resolution proton-density (PD)-weighted imaging as employed in previous studies on human LGN (Giraldo-Chica and Schneider, 2018; Mcketton et al., 2014; Viviano and Schneider, 2015). We then defined the LGN subdivisions using MTV fraction data and the anatomically known volume ratio of the $M$ and $P$ subdivisions. Finally, we tested the validity of the definition based on (1) comparisons with histology data, (2) fMRI measurements of stimulus selectivity, and (3) an analysis of the test-retest reliability.

Using our newly developed method, we found a gradual change in the MTV fraction within the LGN along each axis (lateral-medial, dorsal-ventral, and anterior-posterior). This pattern of change was consistent among subjects, enabling the parcellation of the LGN into two subdivisions in a consistent manner with post-mortem human data. Moreover, the difference in stimulus selectivity of the BOLD response between the subdivisions identified by MTV was consistent with previous physiological studies. The MTV-based LGN parcellation was robust over measurements taken on different days. The parcellation using widely used non-quantitative methods such as the T1 weighted/T2 weighted $(\mathrm{T} 1 \mathrm{w} / \mathrm{T} 2 \mathrm{w})$ ratio map was not sufficient to identify the LGN subdivisions compared with MTV, suggesting that quantitative structural mapping is crucial to identifying the $M$ and $P$ subdivisions in human LGN. This study provides a novel method of non-invasively investigating the properties of LGN subdivisions in living human brains, 
which can be combined with functional or behavioral experiments to test neuroscientific or clinical hypotheses.

\section{Materials and Methods}

\section{Subjects}

Fifteen healthy volunteers (7 females; mean age, 23.53 years; standard deviation, 1.71 years; range, 21-26 years) participated in the study. All of the subjects had normal or corrected-to-normal vision with no clinical history of eye disease. All subjects provided written informed consent to take part in this study. The study was conducted in accordance with the ethical standards of the Declaration of Helsinki and approved by the local ethics and safety committees at the Center for Information and Neural Networks (CiNet), National Institute of Information and Communications Technology (NICT).

\section{Structural MRI data acquisition}

All MRI data were collected at the CiNet using a 3T MAGNETOM SIEMENS Prisma scanner (Siemens Healthcare, Erlangen, Germany) with a 32-channel head coil.

\section{$\underline{T 1 \text { weighted MRI data acquisition }}$}

T1 weighted magnetization prepared-rapid gradient echo (MP-RAGE) images (voxel size, $0.75 \mathrm{~mm} \times 1.0 \mathrm{~mm} \times 0.75 \mathrm{~mm}$; repetition time [TR], $1900 \mathrm{~ms}$; echo time [TE], $3.58 \mathrm{~ms}$; flip angle, $9^{\circ}$; matrix, $256 \times 256$; in-plane acceleration factor, 2) were acquired from all subjects. These images were used as references on which to co-register the subsequent MRI data (PD weighted, MTV, and FMRI data) in the same coordinate space for each individual subject. The total time for the T1 weighted MRI data was approximately $15 \mathrm{~min}$ for each subject.

\section{Proton density (PD)- weighted MRI data acquisition}

PD weighted images were acquired from all subjects to locate the LGN. Acquisition parameter of PD weighted images followed those used in a previous study (Viviano and Schneider, 2015; voxel size, $0.75 \mathrm{~mm} \times 0.75 \mathrm{~mm} \times 1.0 \mathrm{~mm}$; TR, $3000 \mathrm{~ms}$; TE, $21.0 \mathrm{~ms}$; flip angle, $120^{\circ}$; matrix, $256 \times 256$; in-plane acceleration factor, 2). These images were acquired at least 40 times in all subjects. To improve the signal-to-noise ratio, we then continued to repeat the PD-weighted image acquisition if the subjects agreed (maximum number of repetitions: 60 ). Each image consisted of 50-60 coronal slices (slice thickness, $1 \mathrm{~mm}$; no gap) covering the whole posterior thalamus. The total acquisition time for the 
PD weighted MRI data was approximately 60-90 min for each subject, depending on the number of repetitions.

\section{MTV data acquisition}

The MTV data were acquired from all subjects according to a previously described protocol (Mezer et al., 2013; Minami et al., 2020; Oishi et al., 2018; Takemura et al., 2019). Four fast low-angle shot (FLASH) images were measured with flip angles of $4^{\circ}$, $10^{\circ}, 20^{\circ}$, and $30^{\circ}$ (TR, $12 \mathrm{~ms}$; TE, $2.43 \mathrm{~ms}$ ) with $1 \mathrm{~mm}$ isotropic voxels. For the purposes of removing field inhomogeneities, five additional spin echo inversion recovery (SEIR) scans were also measured with an EPI readout (TR, 3 s; TE, 49 ms; $2 \times$ acceleration). The inversion times were 50, 200, 400, 1200, and $2400 \mathrm{~ms}$. The in-plane resolution and slice thickness of the additional scan were $2 \times 2 \mathrm{~mm}^{2}$ and $4 \mathrm{~mm}$, respectively. The total acquisition time for MTV data was approximately $35 \mathrm{~min}$ for each subject. For 11 subjects, we acquired MTV data again on a different day to evaluate the test-retest reproducibility.

\section{T1w/T2w MRI data acquisition}

We also acquired data from 13 subjects for a T1w/T2w ratio map, a technique widely used in the analysis of Human Connectome Project data (Glasser and Van Essen 2011). The T1 weighted image was acquired using a 3D MP-RAGE (TR, $2400 \mathrm{~ms}$; TE, $2.06 \mathrm{~ms}$; $\mathrm{TI}, 1000 \mathrm{~ms}$; flip angle, $8^{\circ}$; bandwidth, $220 \mathrm{~Hz} /$ pixel; echo spacing, $7.5 \mathrm{~ms}$; field of view, $256 \mathrm{~mm} \times 256 \mathrm{~mm} \times 176 \mathrm{~mm}$; matrix, $256 \times 256 \times 176$; voxel size, $1 \mathrm{~mm}$ isotropic resolution) sequence. The T2 weighted image was acquired using sampling perfection with application optimized contrast using different angle evolutions (SPACE: TR, 3200 ms; TE, $438 \mathrm{~ms}$; flip angle, $120^{\circ}$; bandwidth, $574 \mathrm{~Hz} /$ pixel; echo spacing, $3.88 \mathrm{~ms}$; turbo factor, 139; field of view, $256 \mathrm{~mm} \times 256 \mathrm{~mm} \times 176 \mathrm{~mm}$; matrix, $256 \times 256 \times 176$; voxel size, $1 \mathrm{~mm}$ isotropic resolution) sequence. While these acquisition protocols aimed to follow those used in the Human Connectome Project (Glasser and Van Essen 2011), they were not identical due to hardware differences. These data were collected using prescan normalization to reduce image intensity bias.

\section{Structural MRI data analysis}

\section{T1 weighted MRI data}

The T1 weighted MRI images of individual subjects were interpolated and aligned to the ICBM 152 2009b symmetric template in the MNI152 database (http://www.bic.mni.mcgill.ca/ServicesAtlases/ICBM152NLin2009; isotropic resolution, $0.5 \mathrm{~mm}$; Fonov et al., 2011, 2009) using a rigid-body transformation implemented in the FSL FLIRT tool. No spatial smoothing or normalization was performed. The T1 weighted 
MRI image aligned onto the MNI152 space was used for subsequent comparisons between MTV, fMRI, and histological data from individual brains in MNI coordinates.

\section{$\underline{P D \text { weighted MRI data }}$}

The PD weighted image from the first scanning session was used as a reference, and all subsequent PD images were co-registered to the reference using a rigid-body transformation implemented in FSL. We then averaged all of the PD weighted images aligned to the first PD weighted image. The resulting averaged PD weighted image was then interpolated and aligned to the T1 weighted MRI data, which was aligned with the $\mathrm{MNI}$ coordinates at $0.5 \mathrm{~mm}$ isotropic resolution for subsequent analyses.

\section{Quantitative MRI data}

Using the mrQ software package (https://github.com/mezera/mrQ) in MATLAB, the FLASH and SEIR scans were processed to produce the MTV maps (Mezer et al., 2016, 2013). MTV quantifies the macromolecular tissue volume density by estimating a quantitative PD map from the FLASH images after correcting for RF coil bias by the mrQ analysis pipeline using SEIR-EPI scans (Barral et al., 2010; Mezer et al., 2013). Since cerebrospinal fluid (CSF) voxels are entirely filled with water, we assumed that they had a full water volume fraction (WVF). We then calculated the WVF ratio in cortical gray or white matter voxels in comparison with the CSF. The MTV was defined as follows: MTV $=1-W V F$, and this was used to quantify the non-proton macromolecule volume fraction in each voxel. Finally, the MTV map was aligned to the T1 weighted MRI data to enable further comparisons with other images in the same coordinate space. The full analysis pipeline can be found in previous publications (Mezer et al., 2016, 2013; Minami et al., 2020; Oishi et al., 2018; Takemura et al., 2019).

\section{$\underline{T 1}$ w/T2w MRI data}

We obtained a $\mathrm{T} 1 \mathrm{w} / \mathrm{T} 2 \mathrm{w}$ ratio map by co-registering a T2-weighted image to T1-weighted image using the FLIRT tool in FSL (Jenkinson et al., 2002) with six parameters (rigid body) and calculated the ratio between them. The T1w/T2w ratio map was then coregistered to the reference $\mathrm{T} 1$ weighted $\mathrm{MRI}$ data at the $\mathrm{MNI}$ coordinates.

\section{Identifying a LGN region-of-interest from PD-weighted MRI data}

With reference to previous studies (Viviano and Schneider, 2015), we identified the position of the LGN in individual subjects based on the PD weighted image averaged across multiple acquisitions (Figure 1). We manually delineated the whole LGN based on visible intensity differences between the LGN and neighboring tissues (surrounding white matter and CSF) using the ITK-snap tool (http://www.itksnap.org/; Figure 1B). 
Delineation was performed in a series of coronal sections of PD weighted image because the coronal sections have the highest spatial resolution in comparison with the axial and sagittal sections. The whole LGN region-of-interest (ROI) was used in subsequent analyses to classify the $M$ and $P$ subdivisions using MTV.

\section{Parcellation of the LGN based on MTV and other structural MRI maps}

We first rank-ordered all of the voxels within the whole LGN ROI based on their MTV fractions. Previous histological studies have identified that the $\mathrm{P}$ subdivision has a higher neuronal cell density (Hassler, 1966; Yücel et al., 2003, 2000) and greater myelin content (Pistorio et al., 2006) than the M subdivision, and therefore we hypothesized that the $P$ subdivision would demonstrate larger MTV fractions than the M subdivision. Hence, we classified the $20 \%$ of voxels with the lowest MTV fraction as the putative M subdivision and the remaining $80 \%$ of voxels as the putative $\mathrm{P}$ subdivision (Figure $2 \mathrm{~B}$ ). This ratio was based on previously reported volumes of the LGN subdivisions from prior human histological studies (Andrews et al., 1997; Selemon and Begovic, 2007) and was used in a previous fMRI study (Denison et al., 2014). Figure 2 provides examples of MTVbased LGN parcellation in representative hemispheres.

Next, we attempted to parcellate the LGN based on the image intensity of the nonquantitative structural MRI maps (PD weighted image and T1w/T2w ratio map). For the PD-weighted image, we classified $20 \%$ of voxels with the highest image intensity as the putative $\mathrm{M}$ subdivision and the remaining $80 \%$ of voxels as the putative $\mathrm{P}$ subdivision, since the image contrast of PD weighted images demonstrates an opposite trend to that of MTV maps. For the T1w/T2w ratio map, we used the identical criteria as employed for the MTV mapping.

\section{Histological data (BigBrain) analysis}

To compare the MTV-based parcellation of the LGN subdivisions with the histological definition, we analyzed the publicly available BigBrain data (100 $\mu \mathrm{m}$ version of the BigBrain 3-D Volume Data Release 2015 in MNI space from https://bigbrain.loris.ca; Amunts et al., 2013). In brief, BigBrain is a 3D reconstruction of 7404 histological sections of one post-mortem human brain that provides high-resolution anatomical data aligned with MNI coordinate space. In this database, all of the six layers in the human LGN are visible (see Figure 2-S3).

Manual segmentation of the $M$ (layers 1-2) and $P$ subdivisions (layers 3-6) of the human LGN was carried out on the BigBrain data. The manual segmentation was performed by 
a rater who was not an author of this work and unaware of the purpose of this study. We used this $M$ and $P$ subdivision definition from BigBrain as a reference with which to compare the MRI-based parcellation. An example of manual segmentation of the BigBrain data is shown in Figure 2-S3.

\section{Comparison between MRI data with histological data}

To quantify the spatial organization of the $M$ and $P$ subdivisions, we calculated the spatial centers of both subdivisions in MRI and histological data, following the analysis used in a previous fMRI study (Denison et al., 2014). The 3D spatial centers of the $M$ and $P$ subdivisions were defined as the mean voxel coordinates in each spatial dimension (anterior-posterior, dorsal-ventral, and left-right) in MNI space. When co-registering the reference ( $\mathrm{T} 1$ weighted) image to $\mathrm{MNI}$ space, the human thalamic nuclei showed individual differences in their positions, volume, and shape (Csernansky et al., 2004). Therefore, the position of the LGN and its subdivision in the MNI coordinates is variable among individual brains. Thus, we calculated the relative position of the center of each LGN subdivision with respect to the widths of the LGN in each spatial dimension to compare the LGN subdivisions between datasets and brains (Denison et al., 2014).

\section{Functional MRI data acquisition}

All subjects took part in an additional fMRI experiment to investigate the stimulus selectivity of BOLD response in LGN voxels. We acquired $\mathrm{fMRI}$ data with $1.5 \mathrm{~mm}$ isotropic voxels for 10 subjects (S1-S10) and $2 \mathrm{~mm}$ isotropic voxels for 5 subjects (S11S15).

Acquisition parameters. The fMRI data were acquired with an interleaved T2* weighted gradient echo sequence at an isotropic voxel size of 1.5 or $2.0 \mathrm{~mm}$ isotropic by using simultaneous multi-slice EPI sequences (TR, $2250 \mathrm{~ms}$; TE, $40 \mathrm{~ms}$; flip angle, $75^{\circ}$; inplane field of view, $192 \times 192 \mathrm{~mm}$ ) provided by the Center for Magnetic Resonance Research, Department of Radiology, University of Minnesota (https://www.cmrr.umn.edu/multiband/; Moeller et al., 2010). Transverse axial slices (57 slices for $1.5 \mathrm{~mm}$ isotropic voxels and 50 slices for $2.0 \mathrm{~mm}$ isotropic voxels) with no gaps were oriented to cover the LGN and occipital lobe. Certain parameters differed for the acquisition of $1.5 \mathrm{~mm}$ and $2 \mathrm{~mm}$ isotropic voxels acquisitions (multi-band factor, 3; acquisition matrix, $128 \times 128$; echo spacing, $0.93 \mathrm{~ms}$; partial Fourier, 6/8 for $1.5 \mathrm{~mm}$ isotropic voxels; multi-band factor, 2; acquisition matrix, $96 \times 96$; echo spacing, $0.68 \mathrm{~ms}$; partial Fourier was not applied for $2 \mathrm{~mm}$ isotropic voxels), while other parameters were identical. 
Stimuli, block design, and task. All visual stimuli were generated using Psychtoolbox 3 in MATLAB (http://psychtoolbox.org/). Stimuli were projected from a projector (WUX5000, Cannon) located outside the scanner room and reflected via a mirror onto a gammacorrected translucent screen positioned over the subject's head. Gamma-correction was applied using Mcalibrator2 (Ban and Yamamoto, 2013; https://github.com/hiroshiban/Mcalibrator2). Stimuli were presented on a full flat screen $(416 \mathrm{~mm} \times 222 \mathrm{~mm}$ ) at a spatial resolution of $1920 \times 1200$ and a frame rate of $60 \mathrm{~Hz}$. The screen was viewed via a mirror mounted over the subject's eyes. The viewing distance and visual angle of the screen was $92 \mathrm{~cm}$ and $41.2^{\circ} \times 25.8^{\circ}$, respectively.

We adapted two types of stimuli (M-type and P-type stimuli; Figure 4A) designed to elicit selective BOLD responses in the $M$ and $P$ subdivisions (Denison et al., 2014; https://github.com/racheldenison/MPLocalizer). The M-type stimulus was a $100 \%$ contrast, black-white grating with low spatial frequency ( 0.5 cycles per degree) and higher flicker frequency $(15 \mathrm{~Hz})$. The P-type stimulus was a near-isoluminant red-green grating with higher spatial frequency (2 cycles per degree) and lower flicker frequency (5 $\mathrm{Hz})$. The orientation of the grating $\left(0^{\circ}, 30^{\circ}, 60^{\circ}, 90^{\circ}, 120^{\circ}\right.$, or $\left.150^{\circ}\right)$ was changed every $3 \mathrm{~s}$ in a random manner. Prior to the fMRI experiment, we adjusted the luminance of the P-type stimuli to make it perceptually isoluminant using a flicker method (Ives, 1912; Minami and Amano, 2017).

We used a block design for the fMRI experiment, in which each run comprised 15 blocks (six blocks for each of the M- and P-type stimuli and three blocks with a blank screen). Each block was $20.25 \mathrm{~s}$ in duration (including $18 \mathrm{~s}$ for stimulus presentation and $2.25 \mathrm{~s}$ for the blank period, during which the subjects provided their responses). During each block, subjects were instructed to count the number of randomly presented targets, twodimensional Gaussian contrast decrements within the stimuli, while maintaining fixation. During the blank period, the subjects reported how many targets they had seen during the previous stimuli block by pressing a button. The subjects completed 7-8 runs. This procedure and other details of the stimuli, task, and block design have been described previously (Denison et al., 2014).

\section{Functional MRI data analysis}

The fMRI data were analyzed using mrVista (https://github.com/vistalab/vistasoft). We registered the data onto $\mathrm{T} 1$ weighted MRI data to enable comparisons with other MRI datasets. We corrected the slice timing to match the multi-slice acquisition order. The data were then corrected for the subject's motion within and between scans. We fitted a general linear model consisting of predictors ( $\mathrm{M}$ - and P-type stimuli were regressors) 
convolved with the hemodynamic response function (HRF; Boynton et al., 1996) to the time course of each voxel. We used the Boynton HRF to match the procedure in a previous fMRI study (Denison et al., 2014). By fitting the HRF model to the time series of BOLD responses, we estimated the beta values for the $\mathrm{M}$ - and P-type stimuli. We then calculated the difference between them (betam-P) as follows:

beta $_{\mathrm{M}-\mathrm{P}}=$ beta $_{\mathrm{M}}-$ betap $_{\mathrm{P}}$

We used betam-P as an index for evaluating the stimulus selectivity of the LGN subdivisions identified by MTV. We averaged the betam-P across all voxels in each LGN subdivision parcellated on the basis of MTV maps. Finally, we compared betam-P between the MTV-based $M$ and $P$ subdivisions to evaluate the consistency between the stimulus selectivity of the BOLD responses and MTV-based parcellation of the LGN subdivisions.

\section{Test-retest reliability analysis}

To assess the reproducibility of the MTV measurements and MTV-based LGN parcellation, we re-measured MTV of 13 subjects (mean age, 23.85 years; five females). The data acquisition and analysis of MTV retest data were identical to those of the main experiment. We evaluated the reproducibility of the MTV measurements within the LGN by calculating the inter-voxel correlation coefficient $(R)$ between the test and retest data. In addition, we quantified the reproducibility of the MTV-based LGN parcellation by calculating the proportion of LGN voxels classified into the same subdivisions using the test and retest data. We evaluated the statistical significance of this proportion by comparing with a null distribution, which was obtained by shuffling the labeling of the $M$ and $P$ voxels 10,000 times and calculating the distribution of the proportions of voxels classified into the same subdivisions using the test and shuffled data. Finally, we also performed a comparison between the retest and BigBrain data using the same procedure as used for the test data.

\section{Results}

We identified the whole LGN in individual subjects using PD weighted images (Viviano and Schneider, 2015) and then used MTV maps (Mezer et al., 2013) to identify the LGN subdivisions at the single-subject level. The validity of MTV-based parcellation of human LGN was evaluated by comparison with histological BigBrain data (Amunts et al., 2013) and with $\mathrm{fMRI}$ data collected from identical subjects. Furthermore, we tested the validity 
of LGN parcellation data obtained from other types of structural MRI images. Finally, we evaluated the test-retest reliability of the MTV-based parcellation of the human LGN.

\section{The LGN in PD weighted images}

In each individual hemisphere, the position and shape of the whole LGN was visible in the PD weighted images (Figure 1A) as reported previously (Viviano and Schneider, 2015). We delineated the whole $L G N$ in all individual hemispheres by manually inspecting the PD weighted images (Figure 1A, B; see Materials and Methods). Figure 1-S1 depicts the volume of whole LGN in all individual hemispheres, while the LGN volume identified from BigBrain histological data (Amunts et al., 2013) and previous structural MRI studies (Giraldo-Chica and Schneider, 2018; Mcketton et al., 2014) are also shown. Among the 15 subjects tested in this study, the mean ( \pm s.e.m.) volume of the whole LGN was $153.48 \mathrm{~mm}^{3} \pm 2.32 \mathrm{~mm}^{3}$ and $158.40 \mathrm{~mm}^{3} \pm 1.83 \mathrm{~mm}^{3}$ in the left and right hemispheres, respectively. The LGN volume manually identified from PD weighted images was, on the whole, consistent with that obtained from BigBrain data $\left(168.65 \mathrm{~mm}^{3}\right.$ and $162.37 \mathrm{~mm}^{3}$ in the left and right hemispheres, respectively) and previous structural MRI images on the basis of similar PD weighted MRI data (Figure 1-S1; Giraldo-Chica and Schneider, 2018; Mcketton et al., 2014). The right LGN was marginally significantly larger than the left ( $d^{\prime}=0.608, t_{14}=2.136, p=0.0508$, paired $t$-test). Notably, the $\mathrm{M}$ and $P$ subdivisions could not be identified through visual inspections of the PD weighted image.

\section{Identification of $M$ and $P$ subdivisions using the MTV fraction}

Next, we analyzed the MTV maps (Mezer et al., 2013) co-registered with the PD weighted images for each individual subject (Figure 1C). In all of the individual hemispheres, we observed gradual changes in the MTV fractions within the LGN ROIs (Figure 1D); the superior-lateral part of the LGN exhibited a higher MTV fraction than the inferior-medial part. The distribution of MTV did not exhibit clear multiple peaks (Figure 1-S2), which made it difficult to determine a threshold value of MTV at which to separate the $M$ and $P$ subdivisions. The gradual MTV change seen within the LGN was likely due to the limited spatial resolution of the in vivo MTV measurements in this study (voxel size: $1 \mathrm{~mm}$ isotropic). We parcellated the human LGN by incorporating prior knowledge from an anatomical study demonstrating that the area of the $P$ subdivision is roughly four times larger than that of the $M$ subdivision (Andrews et al., 1997). Based on this knowledge, we classified the $20 \%$ of voxels with the lowest MTV as belonging to the putative $M$ subdivision and the remaining $80 \%$ of voxels as belonging to the putative $\mathrm{P}$ subdivisions (Figure 2; see Figure 2-S1 and 2-S2 for results obtained from other 
representative subjects; see Figure 3-S2 for results obtained using a different M/P ratio definition). As expected from the gradual difference in MTV fractions, the voxels classified as part of the putative $\mathrm{M}$ subdivision appeared at the inferior-medial part, whereas those classified as part of the putative $\mathrm{P}$ subdivision appeared the superiorlateral part. We also note that these $\mathrm{M}$ and $\mathrm{P}$ subdivisions were observed as two distinct clusters of voxels which were highly continuous across slices in most hemispheres (Figures 2-S1 and 2-S2).
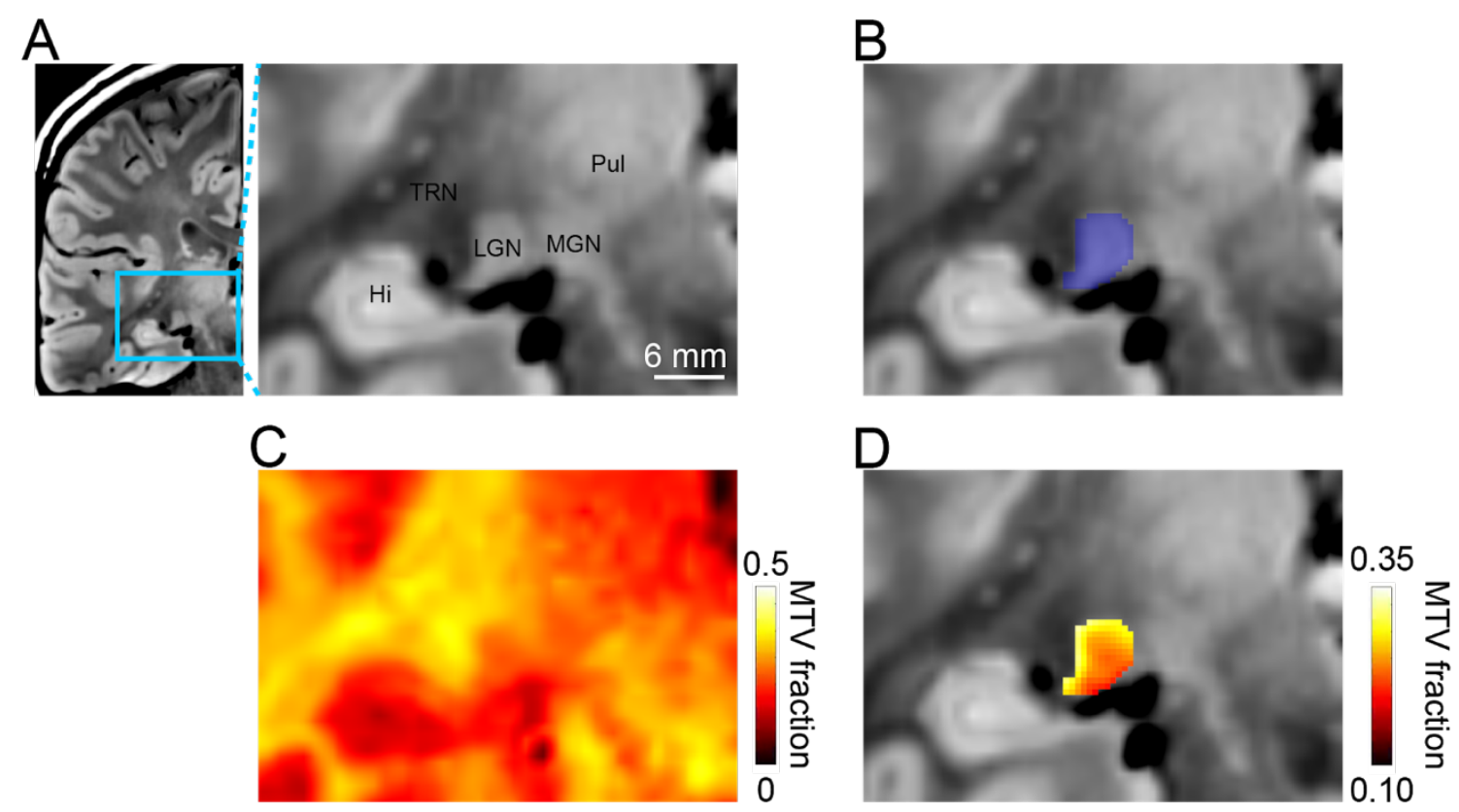

Figure 1. Procedure for identifying the whole LGN and LGN subdivisions in a single human subject

from structural MRI data. (A) A coronal section of a proton density (PD) weighted image in a representative subject (left hemisphere, Subject S10). Left panel, the coronal PD weighted image of the whole left hemisphere. The cyan rectangle indicates the region magnified in the right panel. Right panel, the magnified PD weighted image near the LGN. TRN, thalamic reticular nucleus; Hi, hippocampus; MGN, medial geniculate nucleus; Pul, pulvinar. The scale bar (white line) indicates $6 \mathrm{~mm}$. (B) The ROI covering the whole LGN (translucent blue), which was manually defined from the PD weighted image. (C) Macromolecular tissue volume (MTV) map co-registered with the PD weighted image. The hot color map corresponds to MTV fractions in individual voxels. (D) MTV fractions within the LGN ROI. The MTV fraction gradually changed along the superior-inferior and lateral-medial axes. Note that the scale of the MTV fraction differs from that of panel C. 


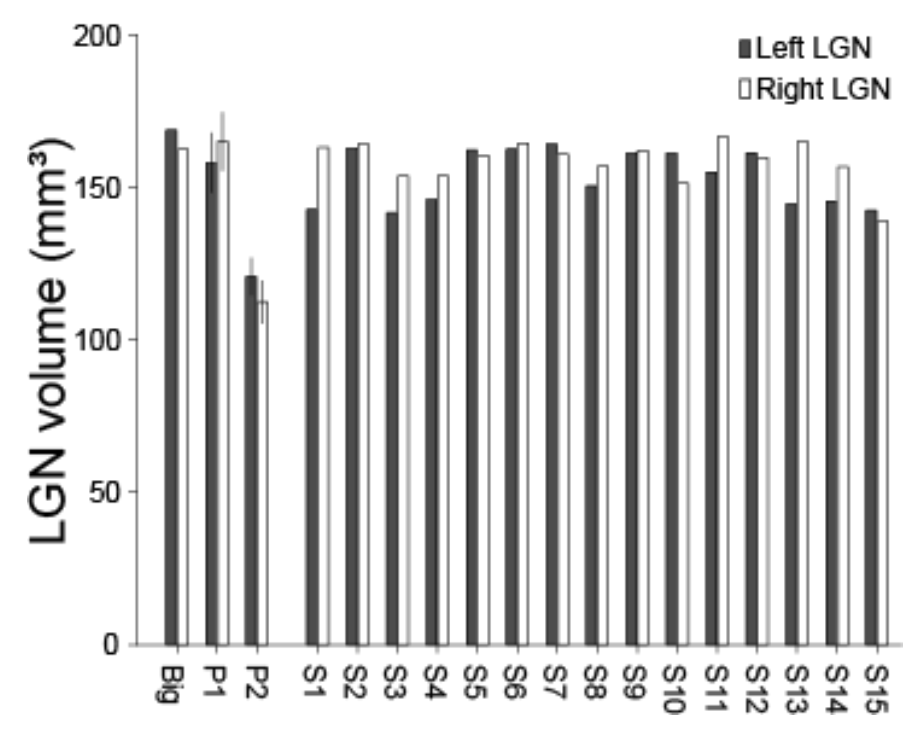

Figure 1-S1: The LGN volume estimated in the current study and previous anatomical and neuroimaging studies. The gray and white bars depict the LGN volumes of the left and right hemispheres, respectively. The LGN volumes in this study $(N=15$, from S1-S15) are within the range of the BigBrain (Big, left end) and previous structural MRI studies (P1, Mcketton et al., 2014 and P2, Giraldo-Chica et al., 2018). We note that both the P1 and P2 data were taken from data collected from healthy control subjects in each paper. Error bars depict \pm 1 S.E.M. 


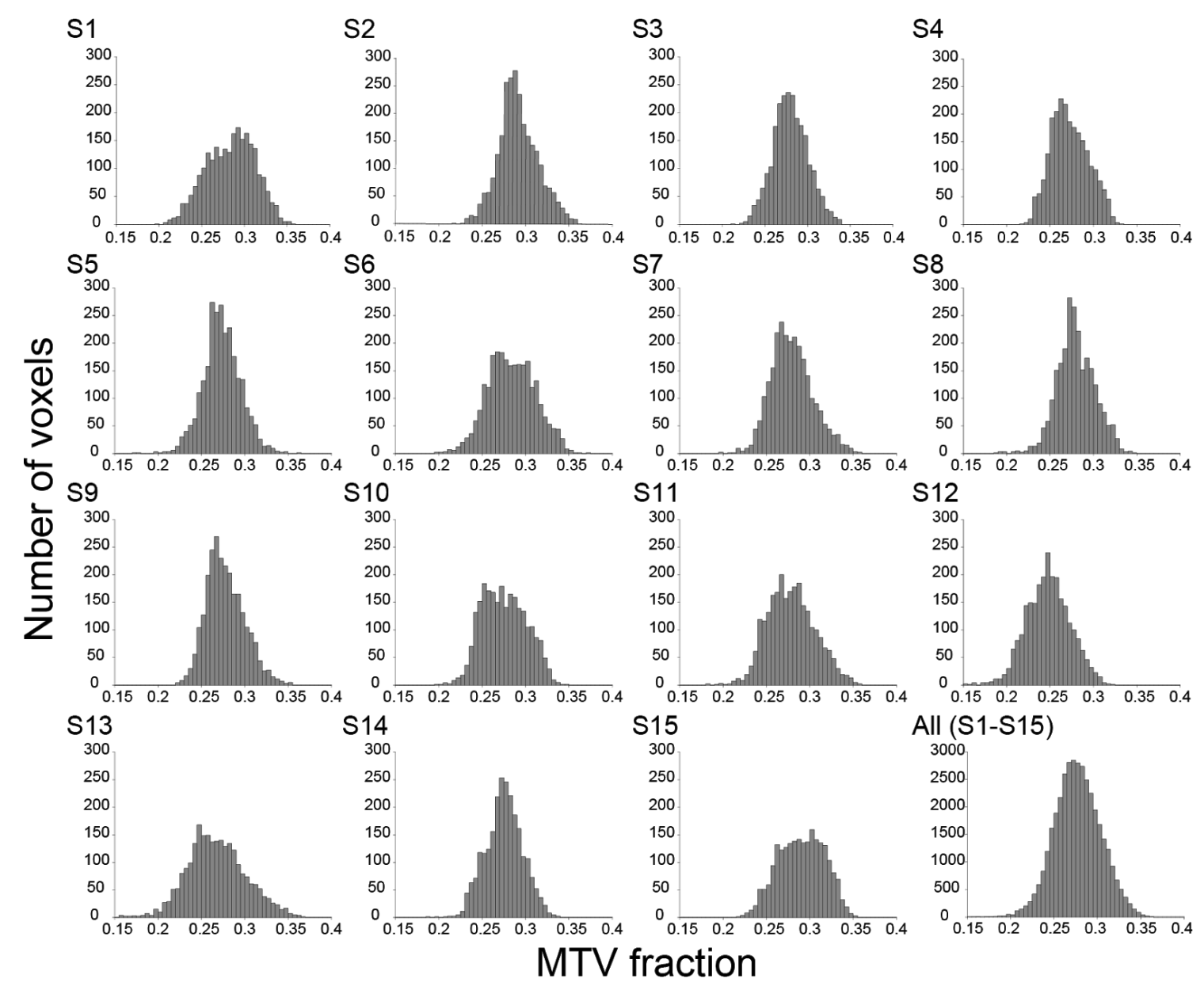

Figure 1-S2: A histogram of the MTV fraction in all LGN voxels for all subjects (S1-S15) and those

pooled across subjects (AlI). The horizontal axis represents the MTV fraction and the vertical axis represents the number of voxels. Bin widths are 0.005 . We did not find a visible bimodal distribution of the MTV fraction within the LGN ROI. 


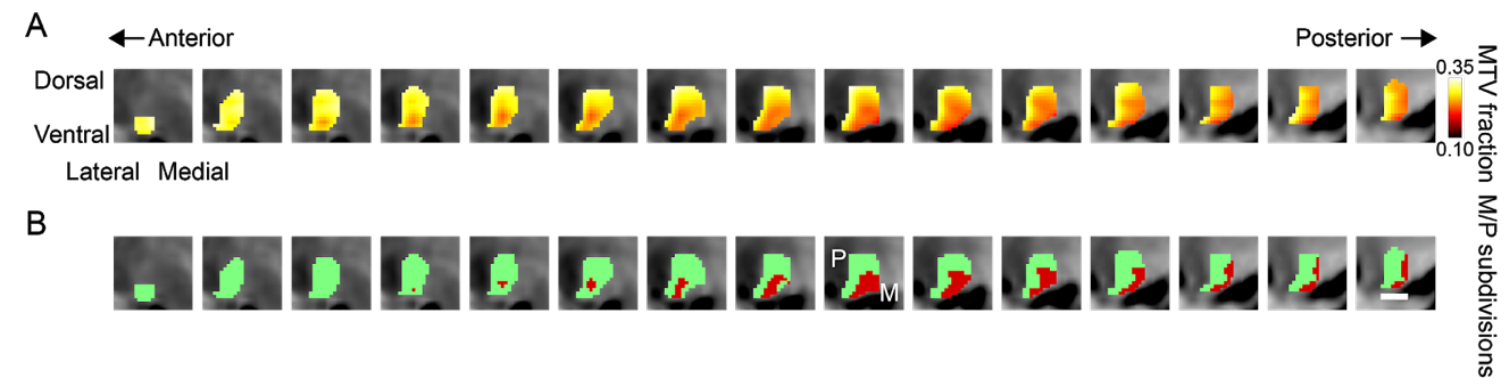

Figure 2. LGN subdivisions parcellated by MTV fraction on a series of coronal sections in a single representative hemisphere. (A) MTV fractions in the LGN ROI overlaid on a series of coronal sections of a PD weighted image (the left and right panels represent anterior and posterior sections; distance between sections: $0.5 \mathrm{~mm}$ ) in a representative hemisphere (left hemisphere, subject S10). The conventions were identical to those used in Figure 1D. (B) The M and P subdivisions estimated from the MTV fractions in the hemisphere shown in panel A. We classified the $20 \%$ of voxels with the lowest MTV fractions as belonging to the $\mathrm{M}$ subdivision (dark red) and the remaining $80 \%$ of voxels as belonging to the $\mathrm{P}$ subdivision (light green). The scale bar indicates $4 \mathrm{~mm}$. 


\section{Left LGN}

S1

$\leftarrow$ Anterior

Posterior $\rightarrow$

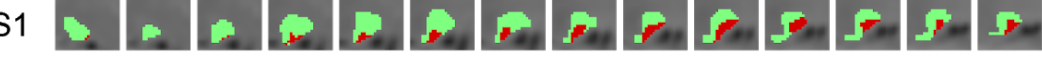

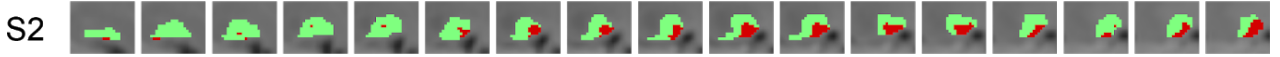

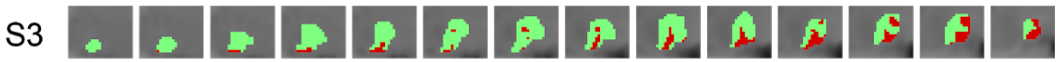

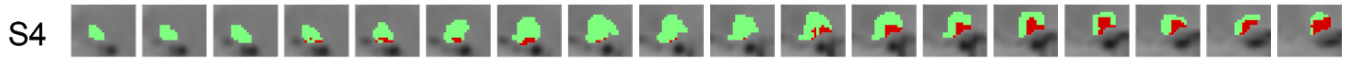

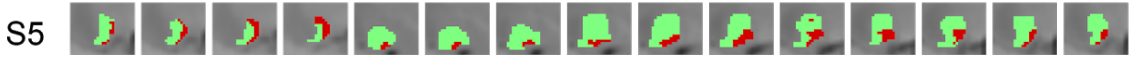

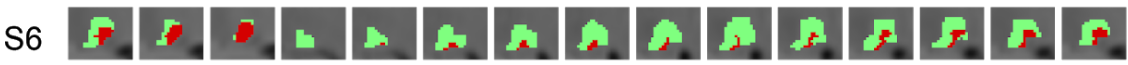

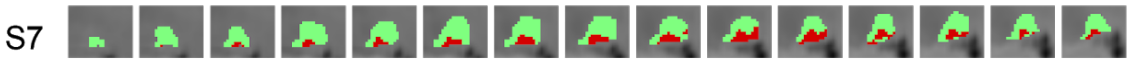

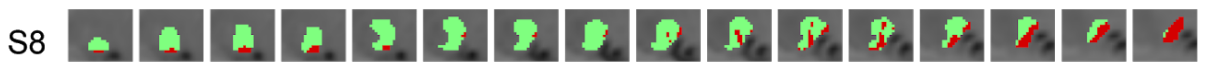

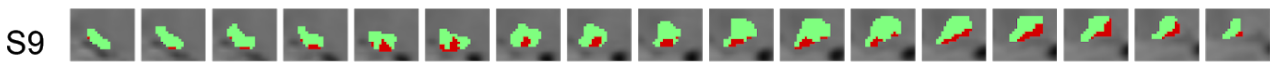

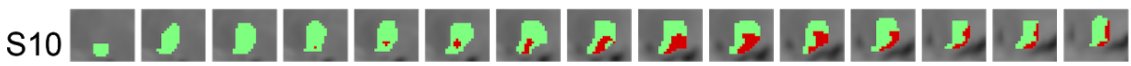

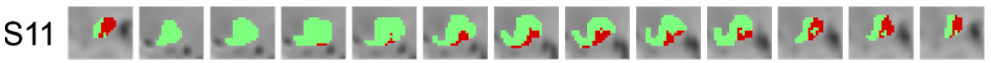

S12 + b +

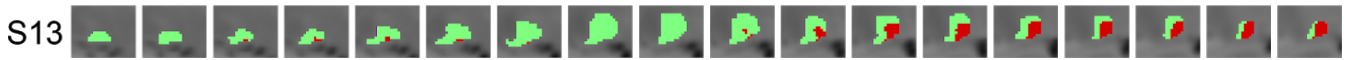

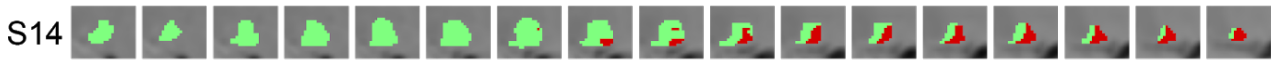

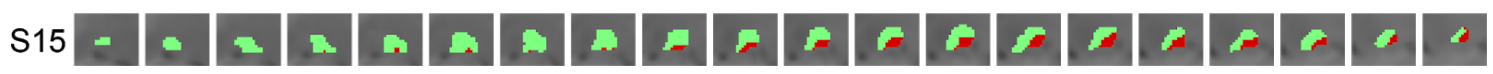

Figure 2-S1: LGN subdivisions parcellated using the MTV in the left hemispheres of all subjects. The

$M$ and $P$ subdivisions identified by the MTV in all subjects $(N=15)$ were overlaid on a series of coronal sections from PD weighted images (left: anterior section; right: posterior section; distance between sections: $0.5 \mathrm{~mm})$. The conventions are identical to those used in Figure 2B. 


\section{Right LGN}

S1 ŁAnterior

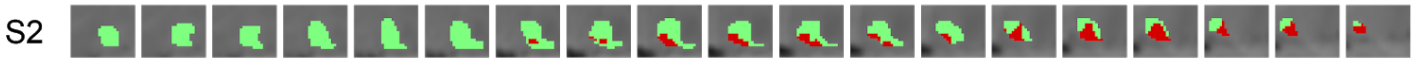

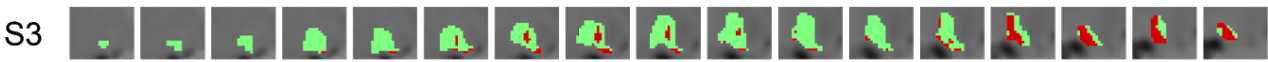

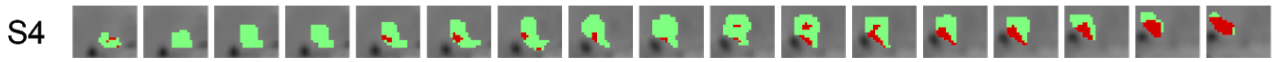

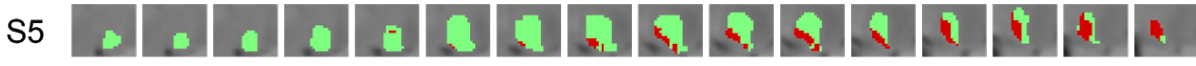

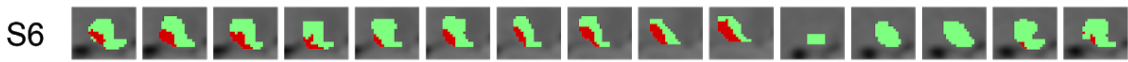

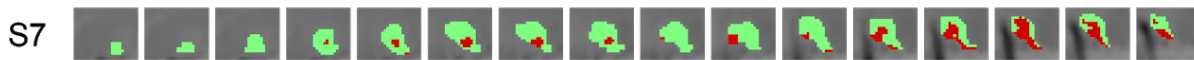

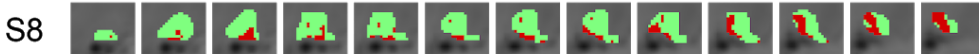

s9

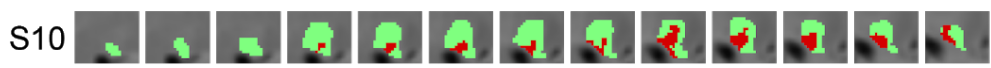

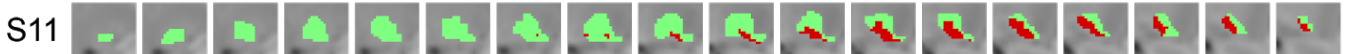

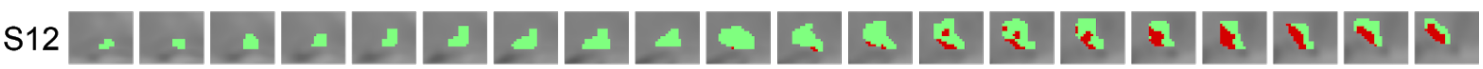

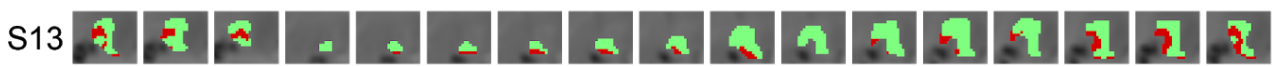

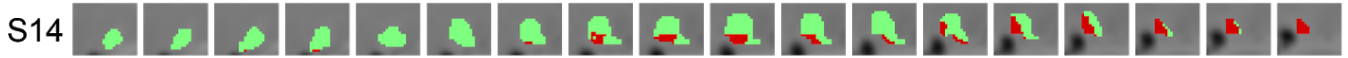

S15 4 a A A A A A A B A

Figure 2-S2: LGN subdivisions parcellated using the MTV in the right hemispheres of all subjects.

The conventions are identical to those used in Figure 2B and 2-S1.

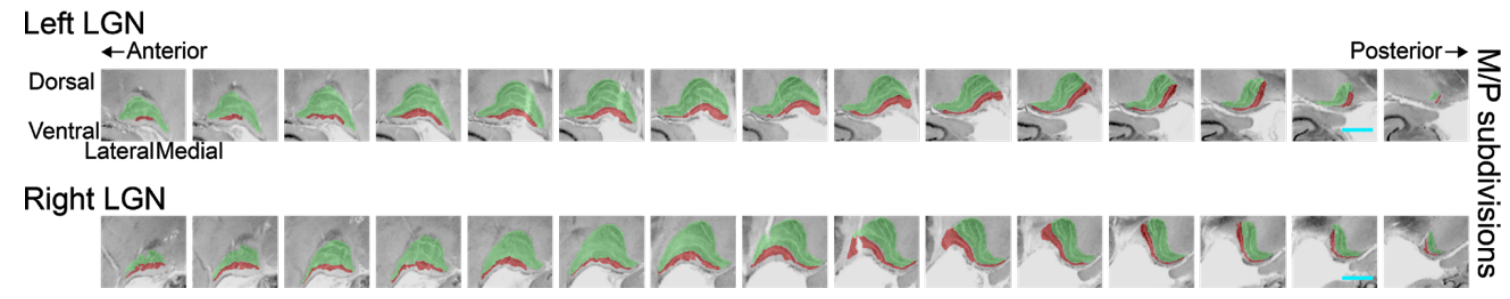

Figure 2-S3: LGN subdivisions on a series of coronal sections from BigBrain data. M (translucent red) and $\mathrm{P}$ subdivisions (translucent green) manually identified from BigBrain data (upper panel, left hemisphere; lower panel, right hemisphere). The cyan scale bar indicates $4 \mathrm{~mm}$, and the distance between the sections was $0.5 \mathrm{~mm}$. All other conventions are identical to those used in Figure 2. 


\section{Validations of $M$ and $P$ subdivisions identified using MTV data}

We evaluated the validity of the MTV-based LGN parcellation by comparing it with the available histological data for human LGN subdivisions (Figure 2-S3). To do so, we calculated the centers of the coordinates among all of the $M$ and $P$ subdivision voxels classified based on the MTV in all hemispheres (Denison et al., 2014) and compared them with those from the BigBrain data (Figure 3; Figure 3-S1). In the BigBrain data, the center of the $M$ subdivision was located in the medial, posterior, and ventral part of the LGN, while the center of the $\mathrm{P}$ division was located in the lateral, anterior, and dorsal part (dashed lines and open circles in Figure 3 and Figure 3-S1). We found that the center of the coordinates for the $\mathrm{M}$ and $\mathrm{P}$ subdivisions defined by the in vivo MTV data showed a similar trend as that seen using the BigBrain data, and this trend was well replicated across all subjects (solid lines and filled circles in Figure 3 and Figure 3-S1). Therefore, these results suggest that the LGN subdivisions identified using the MTV were in good agreement with the anatomical architecture defined using histological human LGN data.

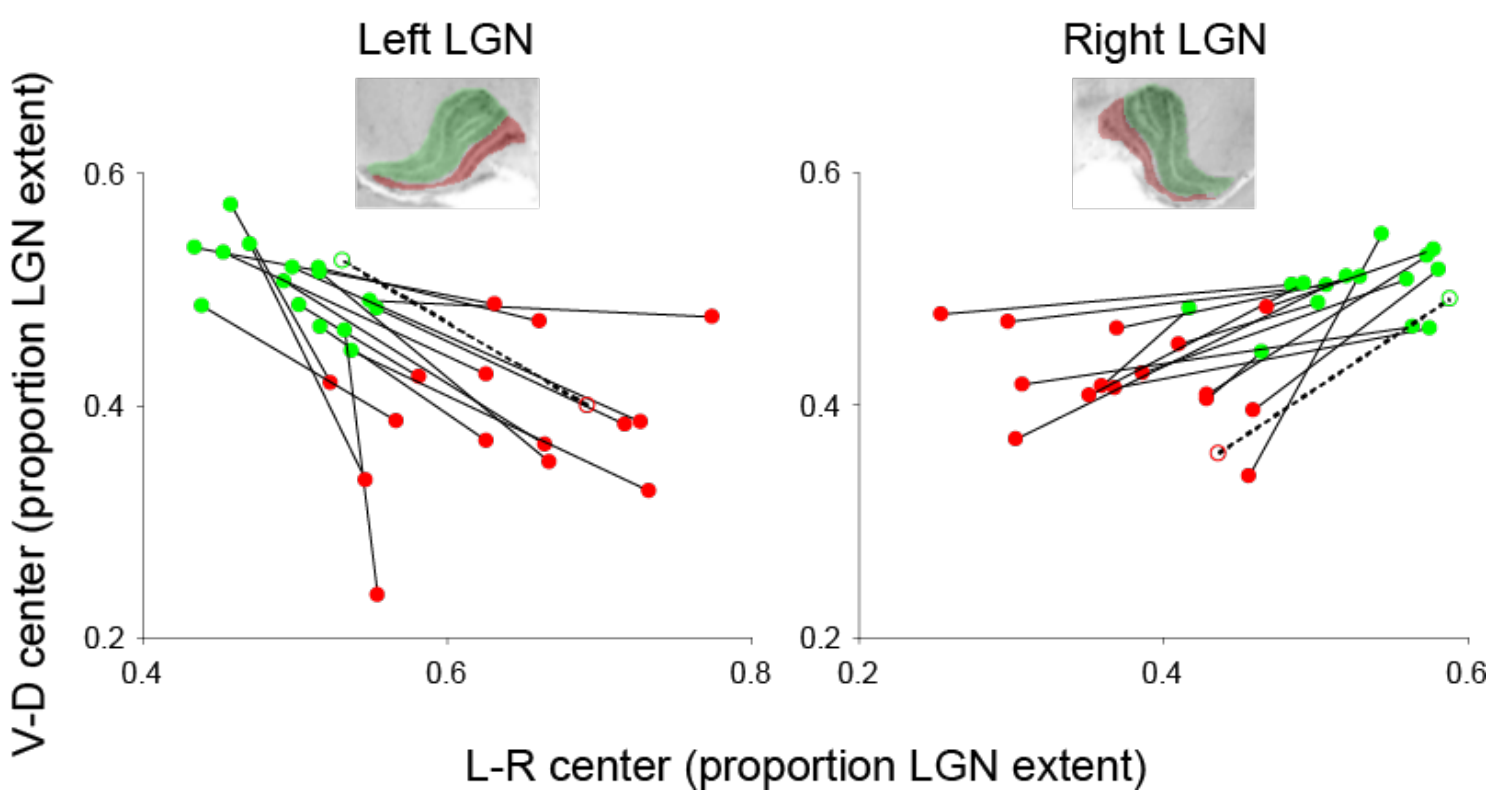

Figure 3: Center positions of the MTV-based M and P subdivisions compared with BigBrain data. The horizontal and vertical axes depict the center positions for the M (red) and P subdivisions (green; left panel, left LGN; right panel, right LGN). The horizontal and vertical axes represent the left-right and ventral-dorsal axes, respectively (Denison et al., 2014). The M and P subdivisions identified in a representative coronal slice using BigBrain data are inserted in each panel. The center positions were calculated in MNI coordinates and are plotted as a proportion of each subject's entire LGN along a given axis. The filled circles and solid lines represent the spatial centers of the $M$ and $P$ subdivisions estimated from the MTV map. The open circles and dotted lines represent the spatial centers of the $M$ and $P$ subdivisions in a human histological 
dataset (BigBrain; Amunts et al., 2013). We found that the centers of the M voxels defined using the MTV were located more medially and ventrally than the $\mathrm{P}$ voxels in all hemispheres, which was consistent with the BigBrain histological data.

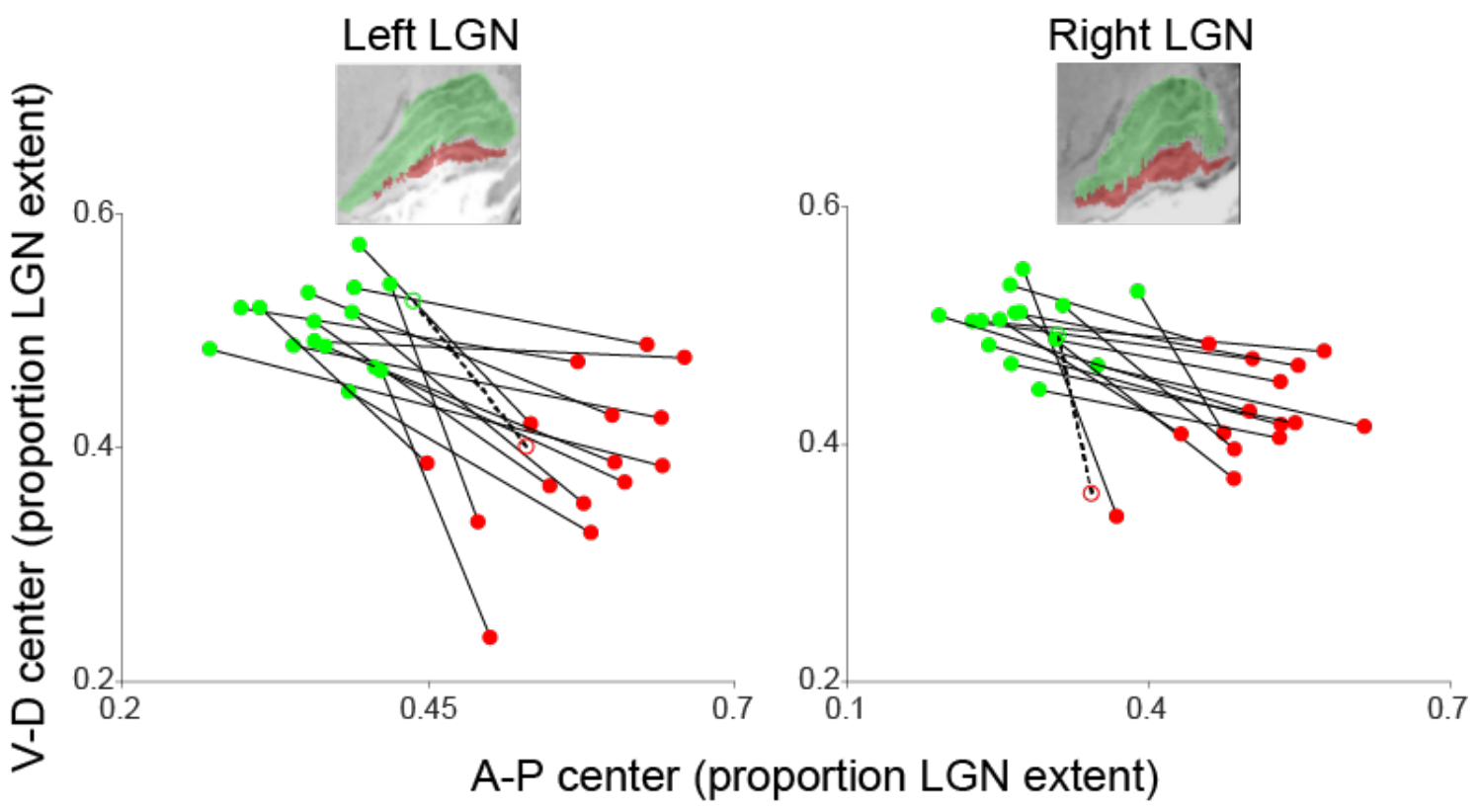

Figure 3-S1: Center positions of the MTV-based M and $P$ subdivisions compared with BigBrain data

in the anterior-posterior/ventral-dorsal axis. The horizontal and vertical axes represent the anteriorposterior and ventral-dorsal axes, respectively. The $\mathrm{M}$ and $\mathrm{P}$ subdivisions identified in a representative sagittal slice using BigBrain data are inserted in each panel. All other conventions are identical to those used in Figure 3.

We replicated the MTV-based parcellation results using two different definitions of the ratio between the $M$ and $P$ subdivisions (i.e., $M: P$ ratio $=19.0: 81.0$ or $28.9: 71.1$ ) to test whether the arbitrary choice of a fixed volume ratio used in the main analysis (1:4; Denison et al., 2014) affected the validity of the MTV-based parcellation. These two ratios correspond to the minimum and maximum proportions of $M$ subdivisions across brains reported in a post-mortem study (Andrews et al., 1997) and determined by our investigations on the BigBrain data. We found that the overall pattern of MTV-based parcellation was well preserved in the current analysis, suggesting that when using a volume ratio from within the range reported by anatomical studies, MTV-based LGN parcellation is consistent with that found using histological data (Figure 3-S2). 


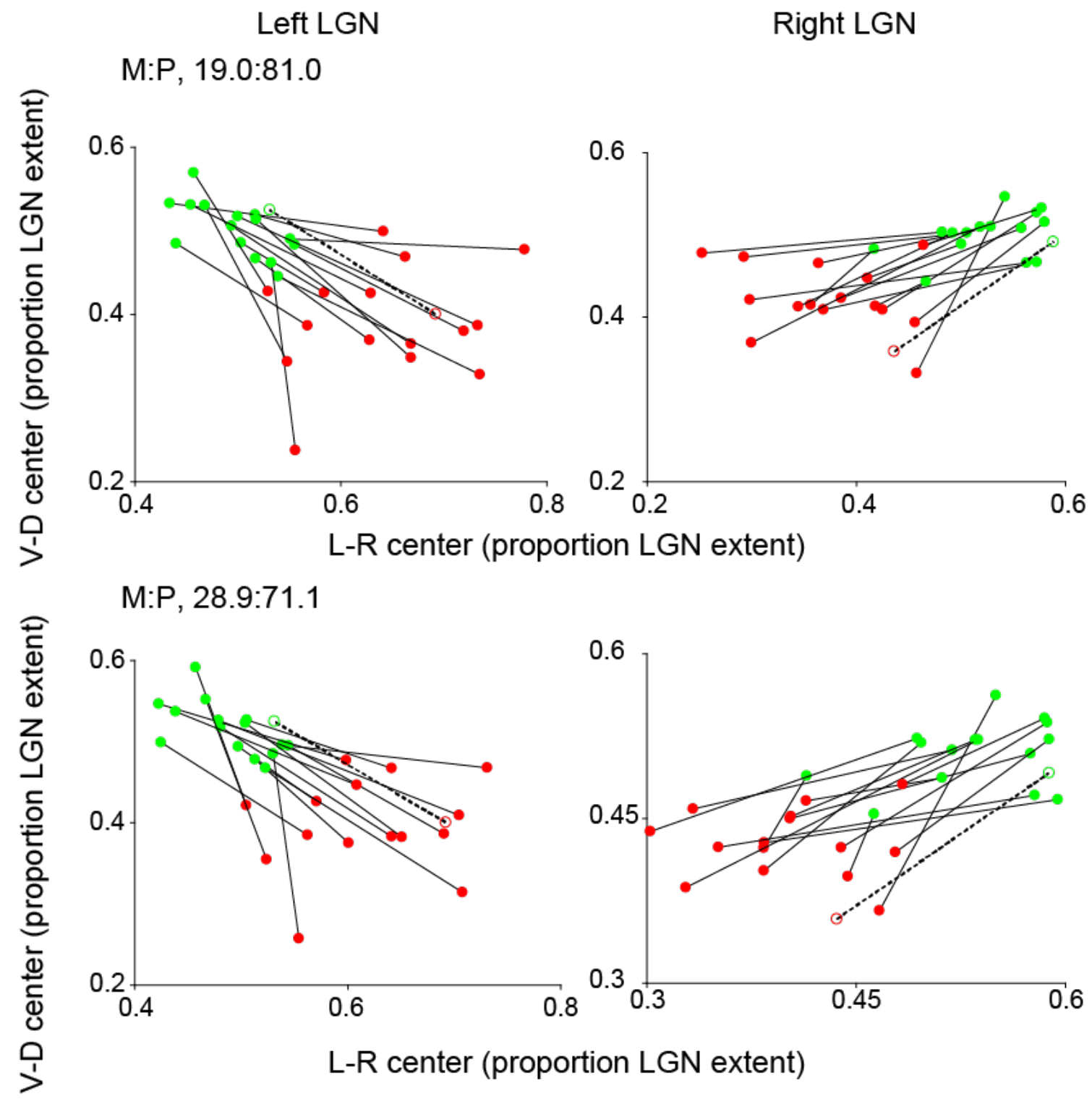

Figure 3-S2: MTV-based parcellation was robust against the ratios of $M$ and $P$ subdivisions. The plots depict the centers of the $M$ and $P$ subdivisions estimated by the MTV fractions with different M:P ratios from the main analysis (top panels, M: 19.0\%; bottom panels, M: $28.9 \%$ ). These two ratios correspond to the minimum and maximum proportions of the $\mathrm{M}$ subdivision across brains reported in a previous post-mortem study (Andrews et al., 1997) and our investigations on the BigBrain data (Amunts et al., 2013). The conventions are identical to those used in Figure 3.

\section{MTV-based $M$ and $P$ subdivisions exhibited different visual stimulus selectivities on fMRI}

Using fMRI, we further tested whether the M and P subdivisions identified using the MTV exhibited the different visual stimulus sensitivities reported in previous macaque and human studies (Denison et al., 2014; Derrington and Lennie, 1984; Usrey and Reid, 
2000; Zhang et al., 2016, 2015). We measured the BOLD responses to a pair of visual stimuli designed to activate the $M$ and $P$ subdivisions differently (Denison et al., 2014; Figure 4A; see Materials and Methods, Functional MRI data acquisition). We examined the extent to which the $M$ and $P$ subdivisions defined using the MTV exhibited different stimulus selectivity in their BOLD response.

We calculated the difference in the beta weights of the M- and P-type stimuli (Beta MstimPStim; a positive value indicated that the BOLD response was greater for the M-type stimuli) for the $M$ and $P$ subdivisions defined using the MTV in individual hemispheres (Figure 4B). A group analysis showed a significant difference in the Beta Mstim-PStim between the $\mathrm{M}$ and $\mathrm{P}$ subdivisions $\left(\mathrm{t}_{14}=4.5416, p=0.0005\right.$ for the left hemisphere; $\mathrm{t}_{14}=$ $3.5838, p=0.0030$ for the right hemisphere, paired $t$-test). This consistency with known stimulus selectivity in the $M$ and $P$ subdivisions further supports the finding that MTVbased parcellation provides reasonable in vivo identification of LGN subdivisions at the level of individual hemispheres. 
A
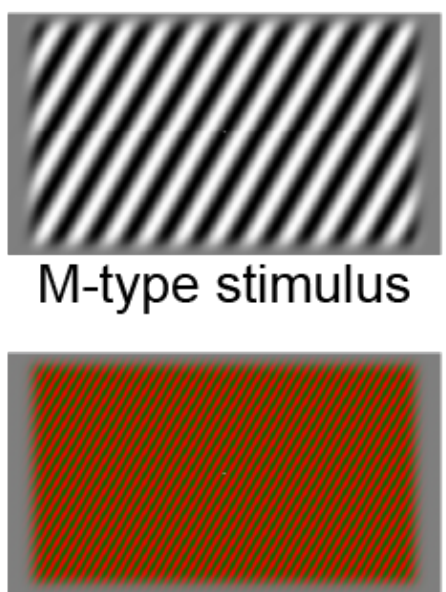

P-type stimulus
B

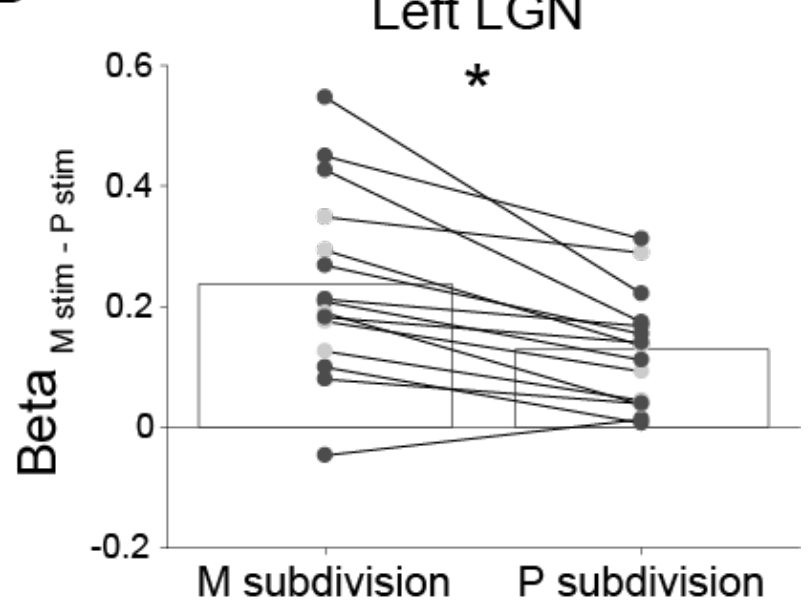

Right LGN

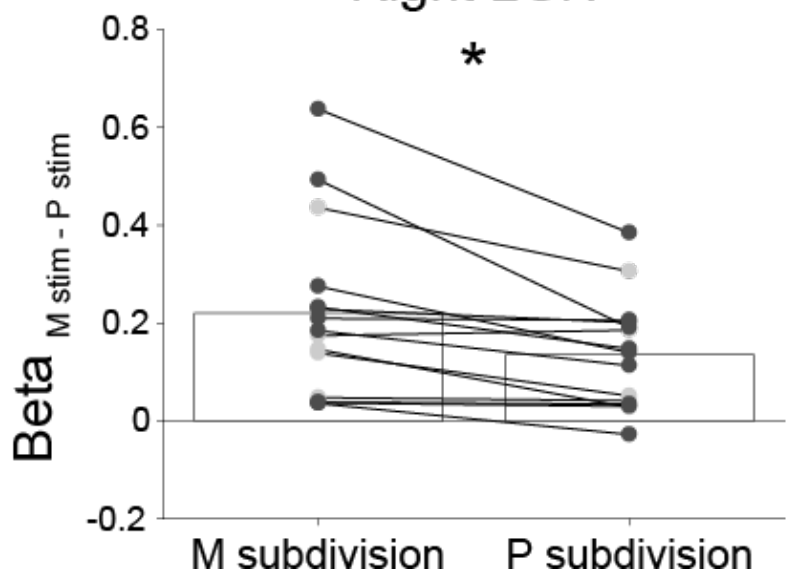

Figure 4: MTV-based $M$ and $P$ subdivisions exhibit stimulus selectivity as reported in previous

literature. (A) Stimuli used to elicit differential BOLD responses from voxels with greater $M$ subdivision representation and those with greater $P$ subdivision representation. Upper panel: An achromatic, low spatial, and high temporal frequency with high luminance contrast grating stimulus used to activate the M subdivision. Lower panel: A high color contrast, high spatial, and low temporal frequency with low luminance contrast grating stimulus used to activate the P subdivision. These stimuli were adapted from Denison et al., (2014). See Materials and Methods for the details of the stimuli. (B) Stimulus selectivity measured by fMRI in the MTV-based M and P subdivisions (top panel, left LGN; bottom panel, right LGN; N = 15 for each). The vertical axis depicts the difference in the beta value between the $M$ - and P-type stimuli (a positive value indicates more selective BOLD responses for the M-type stimuli). The dots indicate data in individual hemispheres. The dark and light gray dots represent the measurements with $1.5 \mathrm{~mm}$ isotropic (S1-S10) and $2.0 \mathrm{~mm}$ isotropic (S11-S15) voxels, respectively. The asterisks represent statistically significant differences in stimulus selectivity between the $\mathrm{M}$ and $\mathrm{P}$ subdivisions measured using the BOLD response (paired $t$-test, *: $p<0.005)$. Details of the fMRI methods are described in Materials and Methods, Functional MRI data acquisition. 


\section{Inter-subject variability and hemispheric differences in MTV fractions}

We examined whether the MTV fractions in the estimated $M$ and $P$ subdivisions were consistent across the healthy subjects who participated in this study (Figure 5). The MTV fractions of the estimated M subdivision were $0.2525 \pm 0.0032$ and $0.2388 \pm 0.0035$ for the left and right hemispheres (mean \pm S.E.M), respectively, whereas the MTV fractions of the estimated $P$ subdivisions were $0.2923 \pm 0.0029$ and $0.2782 \pm 0.0031$ for the left and right hemispheres, respectively. Therefore, the inter-subject variability of the MTV fractions of each subdivision was much smaller than the mean difference between the $\mathrm{M}$ and $\mathrm{P}$ subdivisions in healthy subjects. Given the low variability in the measurements across the healthy population, MTV measurements of the LGN are reliable for use in evaluating how the LGN tissue in patients with, for example, eye disease deviates from that of control subjects.

The MTV of the left LGN was found to be significantly higher than that of the right LGN in both the $M$ and $P$ subdivisions (mean MTV \pm S.E.M for the $M$ subdivision: $0.2525 \pm$ 0.0032 and $0.2388 \pm 0.0035$ in the left and right hemispheres, respectively, $d^{\prime}=1.0708$, $t_{14}=4.9078, p=0.0002$, paired $t$-test; $\mathrm{P}$ subdivision: $0.2923 \pm 0.0029$ and $0.2782 \pm$ 0.0031 in the left and right hemispheres, respectively, $d^{\prime}=1.220, t_{14}=5.1707, p=0.0001$, paired $t$-test). These significant hemispheric asymmetries in the MTV may not be due to measurement biases in the entire image, as no inter-hemispheric MTV differences were observed in the whole gray matter or white matter (mean MTV \pm S.E.M: whole gray matter, $0.2038 \pm 0.0015$ and $0.2033 \pm 0.0013$ in the left and right hemispheres, respectively, d' $=0.0933, t_{14}=0.6404, p=0.5322$; whole white matter, $0.3069 \pm 0.0021$ and $0.3073 \pm 0.0022$ in the left and right hemispheres, respectively, $d^{\prime}=0.0533, t_{14}=$ 1.3652, $p=0.1937$ ). The asymmetry of the MTV suggested that there may be some inter-hemispheric tissue differences in the LGN, although the exact microstructural interpretation of this effect requires further study. 

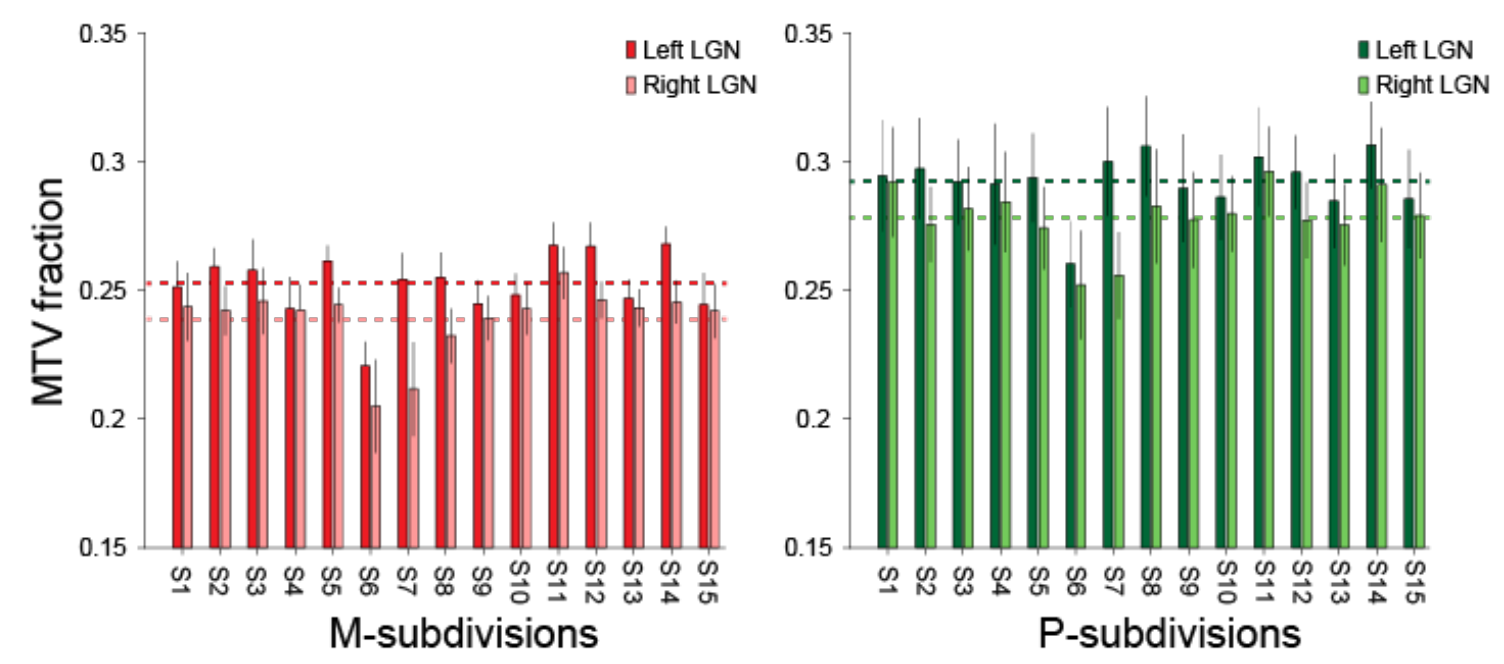

Figure 5: The MTV fractions in the estimated $M$ and $P$ subdivisions were consistent across all healthy subjects. The vertical axis depicts the MTV fraction averaged across voxels within the M (left panel, red) and $\mathrm{P}$ subdivisions (right panel, green) in individual hemispheres. The dark and light bars indicate the MTV fractions of the left and right hemispheres, respectively. The thin dotted lines indicate the averages for each hemisphere across subjects. The MTV fractions in these subdivisions were consistent across 15 healthy subjects. The error bars depict \pm 1 standard deviation across the voxels.

\section{LGN $M$ and $P$ subdivisions were not parcellated from PD weighted images and $T 1 w / T 2 w$ ratio maps}

MTV is a useful method for obtaining quantitative measurements of brain tissue properties (Berman et al., 2018; Duval et al., 2017; Mezer et al., 2013). However, a number of studies have used other types of MRI-based metrics, such as the ratio between $T 1$ weighted and $T 2$ weighted images (T1w/T2w ratio), to evaluate tissue properties. The acquisition time for these images is shorter, but the measurements are not fully quantitative (Glasser et al., 2016; Glasser and Van Essen, 2011). Therefore, we tested whether the $M$ and $P$ subdivisions can be similarly parcellated using image intensities on non-quantitative structural MRI scans to evaluate the potential advantages of the MTV-based approach.

First, we tested LGN parcellation using the intensities of PD weighted images; these were used to identify the whole LGN (Figure 6A). Parcellation was also investigated using the $\mathrm{T} 1 \mathrm{w} / \mathrm{T} 2 \mathrm{w}$ ratio map, which has been used often in previous studies to parcellate cortical areas (Glasser and Van Essen, 2011; Figure 6B). In both cases, we found that coordinates for the centers of the $\mathrm{M}$ and $\mathrm{P}$ subdivisions in the majority of subjects were inconsistent with those obtained using the histological data (BigBrain), suggesting that these maps were not sufficient for parcellating the $M$ and $P$ subdivisions in the $L G N$. This 
most likely occurred because these values are prone to measurement biases (Shams et al., 2019). The unsuccessful parcellation of the $M$ and $P$ subdivisions using the PD weighted images or T1w/T2w maps indicated the advantage of acquiring MTV data for LGN parcellation.

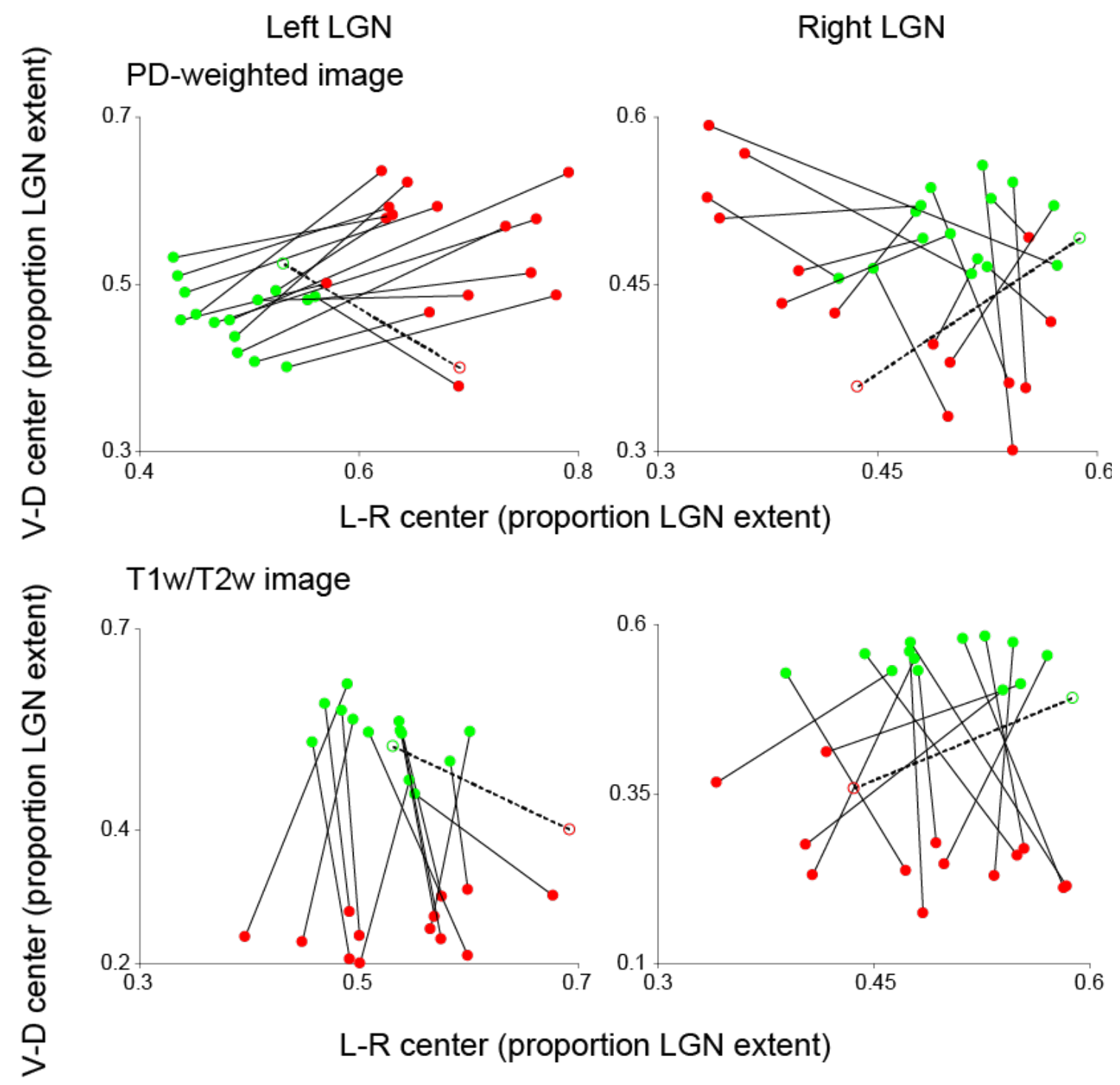

Figure 6: LGN subdivisions may not be identifiable from non-quantitative structural MRI maps. (A)

LGN parcellation based on the image intensities of PD weighted images. The centers of the $M$ and $P$ voxels vary across the hemispheres and are inconsistent with the LGN coordinates obtained using the BigBrain data. (B) LGN parcellation based on the image intensities of T1w/T2w maps. In most hemispheres, the center points along the left-right axis were similar between the $M$ and $P$ subdivisions, unlike those seen using the BigBrain data. The conventions are identical to those used in Figure 3. 


\section{LGN M and $P$ subdivisions were parcellated using the MTV in the retest session}

We tested the test-retest reliability of the $\mathrm{M}$ and $\mathrm{P}$ parcellations by performing the same MTV measurement in 13 subjects on a different day. The MTV fractions of voxels within the LGN ROI were highly correlated between the test and retest experiment $(r=0.78$, Figure 7A). In calculating the probability that individual voxels can be classified into the same subdivisions between the test and retest experiments, we demonstrated that $85.62 \%$ and $82.31 \%$ of voxels in the left and right LGN, respectively, were classified in same subdivision in both the test and retest experiments (Figure 7B; mean across subjects). To assess the statistical significance of these numbers, we randomly classified $80 \%$ of voxels into the P subdivision and the remaining $20 \%$ into the M subdivision to obtain a null distribution. We repeated this process by shuffling the voxels 10,000 times. The maximum probabilities of the voxels being classified into the same subdivisions between the test and shuffled data were $71.73 \%$ and $71.55 \%$ for left and right hemispheres (mean across subjects), respectively, suggesting that the test-retest reliability of MTV-based parcellation was highly significant $(p<0.0001)$. Finally, using the retest dataset, we replicated the results indicating that the centers of the $M$ and $P$ subdivisions showed the same spatial patterns as in the histological data (Figure 7C). Taken together, these results support a considerable degree of reproducibility for our results from the MTV-based LGN parcellation. 
A

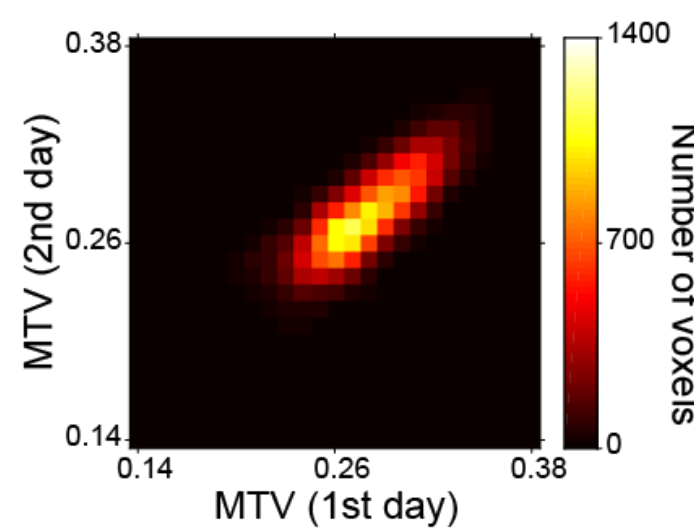

C

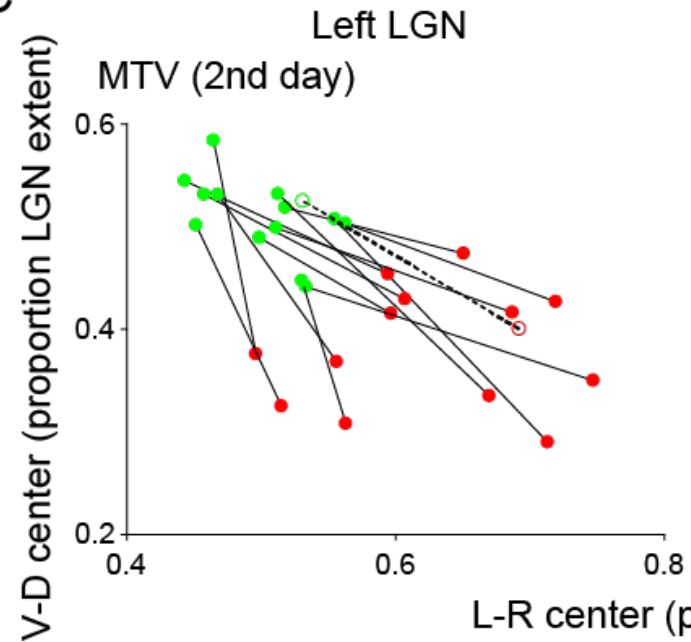

B

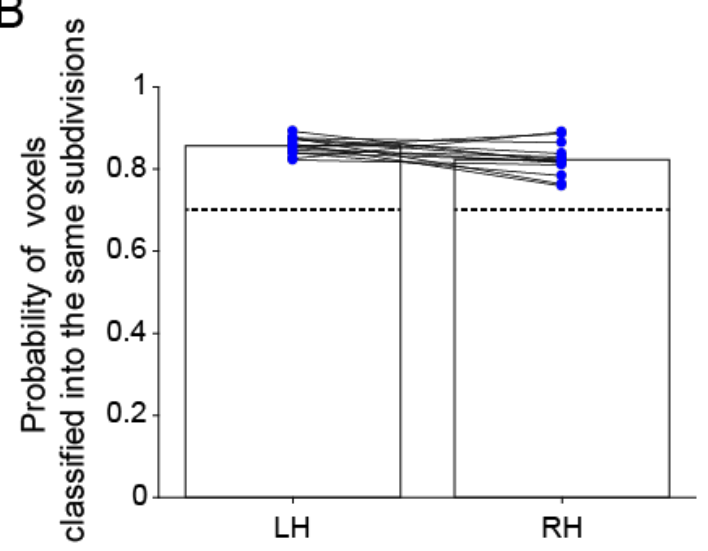

Right LGN

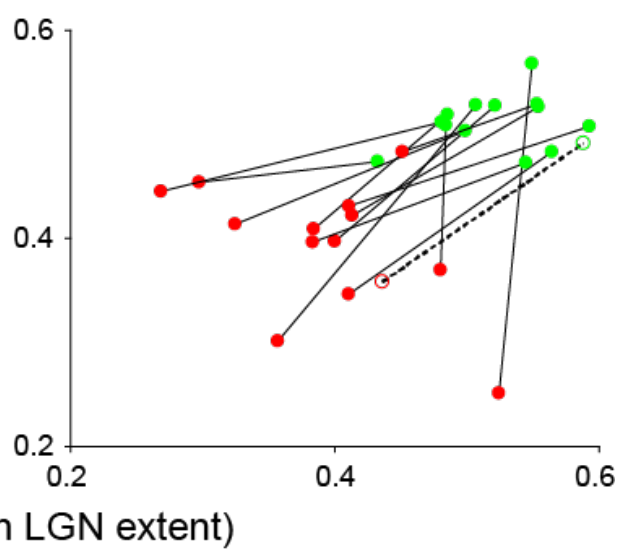

Figure 7: Test-retest reproducibility of MTV-based parcellation. (A) Two-dimensional histogram comparing the MTV measurements across days in LGN voxels. The data are derived from LGN voxels pooled across subjects, who took part in the retest scans $(N=13)$. The color map indicates the number of voxels. The correlation coefficient of MTV measurements across days was $0.78\left(p<10^{-10}\right)$. This high correlation coefficient indicated that the MTV measurements were reproducible across days.

Reproducibility of classification. The vertical axis depicts the probability that individual voxels were classified into the same LGN subdivisions between the test and retest dataset. The individual dots depict the results in individual hemispheres. The dotted lines depict the maximum probability of voxels being classified into the same subdivisions between the test and shuffled data, which was equal to $p=0.0001$. (C) The centers of the coordinates for the $\mathrm{M}$ and $\mathrm{P}$ subdivisions identified with MTV-based parcellation using retest dataset (filled circles and solid lines). We replicated the results on the centers of $M$ and $P$ subdivisions in a consistent manner with those in BigBrain data (Amunts et al., 2013; open circles and dotted line). The conventions are identical to those used in Figure 3. 


\section{Discussion}

It is widely known that the human LGN consists of functionally and anatomically different subdivisions. However, identifying these subdivisions in individual living human brains using conventional structural neuroimaging methods had been challenging. In this study, we demonstrated the approximate parcellation of LGN subdivisions at the single-subject level by combining in vivo structural MRI methods (multiple PD weighted imaging and MTV measurement). The spatial positions of the identified LGN subdivisions were consistent with those identified using histological data (Amunts et al., 2013). Furthermore, using $\mathrm{fMRI}$, we confirmed that these subdivisions exhibit different stimulus selectivity, which is consistent with the findings of previous physiological studies. Finally, we confirmed that MTV-based LGN parcellation is highly consistent across datasets acquired on different days, suggesting that the proposed method is highly reproducible. Other non-quantitative MRI methods did not provide LGN parcellation consistent with histological data. Taken together, this study provides evidence of the utility of this quantitative structural MRI approach to LGN parcellation and establishes methods of measuring the structural properties of human LGN subdivisions in single living human subjects using a clinically feasible $3 \mathrm{~T}$ MRI scanner.

\section{Microstructural origin of MTV-based parcellation}

Our results demonstrated that the MTV fraction can be a useful measurement for distinguishing $M$ and $P$ subdivisions. One might ask what types of microstructural differences lead to MTV differences between M and $P$ subdivisions. In principle, the MTV quantifies non-water macromolecular volumes on the basis of calibrated quantitative PD maps (Mezer et al., 2013). Phantom experiments confirmed that MTV measurements correlate with the lipid fraction (Filo et al., 2019; Mezer et al., 2013; Shtangel and Mezer, 2020). However, there is no established theory on how much MTV variance in a particular brain area can be explained by specific types of microstructural properties. A number of histological studies on non-human primates reported differences in anatomical properties between $\mathrm{M}$ and $\mathrm{P}$ subdivisions, such as the higher neuronal cell density (Hassler, 1966; Yücel et al., 2003, 2000) and greater myelin content in the $P$ subdivision (Pistorio et al., 2006). A recent post-mortem human study confirmed these properties (Müller-Axt et al., 2020). These results are in line with our results showing a larger MTV fraction in the $P$ subdivision than in the $M$ subdivision, since both a larger number of cells and greater myelin content will result in a larger lipid volume fraction. It is also possible that other neurobiological factors such as glial cell density also partly explain the difference in MTV between the M and $P$ subdivisions. This remains an open 
question for future investigations that more directly compare histology and quantitative MRI maps.

In this study, we also determined a significant difference in the MTV fraction between the left and right LGN. To our knowledge, this is the first neuroimaging study to demonstrate this inter-hemispheric difference in human LGN tissue properties. While speculative, these results may be related to those of a previous study showing a difference in the number of neurons in left and right LGN of rhesus monkeys (Williams and Rakic, 1988). Further histological examination will be necessary to establish what type of microstructural differences exist between the hemispheres of the human LGN.

\section{Advantage of MTV-based parcellation over other structural MRI methods}

In standard practice, many neuroimaging studies have utilized T1-weighted and/or T2weighted images to locate cortical areas or subcortical nuclei. While the relative values in these images are useful for identifying the borders between gray and white matter, their absolute values cannot be interpreted as being quantitative, because the measurements are affected by multiple sources of inhomogeneity such as $B_{1}$ transmitter $\left(\mathrm{B}_{1}{ }^{+}\right)$inhomogeneity or coil gain bias. Recent developments in quantitative MRI have enabled the quantification of MRI parameters, which allows the comparison of brain tissue properties between human subjects (Cercignani et al., 2018; Forstmann et al., 2016; Keuken et al., 2017; Mezer et al., 2013; Weiskopf et al., 2015). These quantitative MRI measurements have provided valuable insights into the tissue properties of cortical areas (Lutti et al., 2014; Sereno et al., 2013) and white matter (Stüber et al., 2014; Takemura et al., 2019).

Mezer and colleagues (2013) proposed MTV methods and demonstrated consistency of MTV measurements with lipid volume fractions in a phantom, high test-retest reproducibility, and sensitivity for white matter tissue changes in patients with multiple sclerosis. A strong advantage of this method is its independence from the static magnetic field strength, since it is based on PD measurements calibrated by assuming that the water fraction in CSF voxels is $100 \%$. In fact, Mezer et al. (2013) demonstrated that MTV measurements in the brain are consistent across measurements performed using different types of hardware. Therefore, we chose MTV mapping as a potential method for parcellating the human LGN, since it is relatively independent from hardware choices and thus useful for future clinical studies. 
We found that MTV enabled the parcellation of the LGN in a consistent manner to that seen using histological data (Figure 3) and subdivisions identified by MTV exhibited the stimulus selectivity consistent with previous physiological studies (Figure 4). MTV-based parcellation was superior to parcellation based on non-quantitative MRI maps (PD weighted images or T1w/T2w maps; Figure 6). This is most likely because the MTV was corrected for $\mathrm{B}_{1}{ }^{+}$inhomogeneity, while the other maps were not. While the $\mathrm{T} 1 \mathrm{w} / \mathrm{T} 2 \mathrm{w}$ map has been demonstrated to enhance tissue contrast and thus be useful for delineating borders between brain areas (Glasser and Van Essen, 2011) and has advantages in terms of shorter acquisition time, several studies demonstrated inconsistencies between $\mathrm{T} 1 \mathrm{w} / \mathrm{T} 2 \mathrm{w}$ and quantitative MRI measurements that were more sensitive to myelin (Arshad et al., 2017; Hagiwara et al., 2018; Uddin et al., 2018). These inconsistencies are most likely due to the fact that $T 1 w / T 2 w$ images are not calibrated for $\mathrm{B}_{1}{ }^{+}$ inhomogeneity (Glasser and Van Essen, 2011). While we have not excluded the possibility that ad-hoc $\mathrm{B}_{1}{ }^{+}$bias field correction (Glasser et al., 2013) may improve LGN parcellation using T1w/T2w images, our results demonstrated that MTV-based LGN parcellation performed better than that with $\mathrm{T} 1 \mathrm{w} / \mathrm{T} 2 \mathrm{w}$, most likely because of the superior calibrations for $\mathrm{B}_{1}{ }^{+}$inhomogeneity in the LGN.

\section{Comparison with fMRI-based LGN parcellation}

A few $\mathrm{fMRI}$ studies have examined the spatial pattern of visually-evoked BOLD signals in the LGN (Denison et al., 2014; Zhang et al., 2015). These studies demonstrated that clusters of LGN voxels preferentially respond to distinct types of visual stimuli, which was consistent with neurophysiological findings, suggesting that an approximate identification of the LGN subdivisions in living humans can be achieved using fMRIbased measurements of visual stimulus sensitivities. Quantitative structural MRI-based parcellation methods as shown in this study have several advantages compared with fMRI-based parcellation methods. First, the fMRI-based methods require a precise control of visual stimuli, which involves the presentation of isoluminant stimuli (Denison et al., 2014); this is unnecessary when using the structural MRI-based methods. Furthermore, the use of visual stimuli limits the application of LGN parcellation methods in clinical studies of patients with visual field loss or when using MRI scanners without visual stimulus presentation equipment. Second, structural MRI-based methods are more spatially precise, since the voxel size for MTV measurements (e.g., $1 \mathrm{~mm}$ isotropic in this study) is generally smaller than those used in fMRI experiments (e.g., $1.8 \times 1.8 \times$ $1.5 \mathrm{~mm}$ for 3T or 1.2 1.5 mm isotropic for 7T in Denison et al., 2014). When using fMRI, large veins passing through multiple voxels can limit the spatial specificity of the BOLD signal (Kay et al., 2019; Uludağ and Blinder, 2018). Therefore, our methods are 
advantageous in terms of spatial precision, although fMRI-based methods are sure to improve (Huber et al., 2018, 2014; Kay et al., 2020). Finally, MTV-based parcellation has higher test-retest reliability across days (Figure 7) compared with that reported previously in an fMRI study ( $r<0.4$; Denison et al., 2014). Therefore, MTV-based parcellation provides more stable identification of $\mathrm{M}$ and $\mathrm{P}$ subdivisions in individual living human brains.

\section{Limitations and future directions}

In this study, we classified voxels into $\mathrm{M}$ and $\mathrm{P}$ subdivisions using a fixed volumetric ratio (1:4). However, previous studies reported inter-subject variability in the ratio of the $M$ and $\mathrm{P}$ subdivision volumes in post-mortem human data (Andrews et al., 1997; Hickey and Guillery, 1979). Therefore, it would be more ideal to classify the LGN voxels into M and $P$ subdivisions by fitting a mixture model composed of two curves with distinct peaks to the distribution of the MTV fractions in each individual LGN. However, this approach was not practical in this study as the MTV-based distribution of LGN voxels in our in vivo data did not show two distinct peaks corresponding to the $M$ and $P$ subdivisions (Figure 1S2). Given that a recent study using high-resolution ex vivo quantitative structural MRI (220 $\mu \mathrm{m}$ isotropic) using 7T MRI demonstrated a parcellation of LGN subdivisions using the aforementioned curve fitting procedure (Müller-Axt et al., 2020), we expect that future improvement in the spatial resolution of in vivo quantitative MRI will provide LGN parcellation without assuming a fixed volumetric ratio, enabling quantitative comparisons of $M$ and $P$ subdivisions volumes across subjects.

The other limitation of the MTV-based parcellation method proposed in this work was the relatively long acquisition time. Our method of identification required the acquisition of multiple structural images, each of which required a considerable length of time to obtain (60-90 and $28 \mathrm{~min}$ for the PD weighted image and quantitative structural MRI, respectively) to ensure higher signal quality at the LGN and quantification of the MTV (Giraldo-Chica et al., 2015; Giraldo-Chica and Schneider, 2018; Mcketton et al., 2014; Mezer et al., 2013; Viviano and Schneider, 2015). While the trade-off between signal quality and acquisition time is inherent to any neuroimaging method, prolonged acquisition times limit the application of MTV-based parcellation to studies on clinical populations. Further development of the quantitative MRI method with shorter acquisition times (Caan et al., 2019; Ma et al., 2013; Marques et al., 2010; Warntjes et al., 2008) is essential to increasing the opportunity to measure LGN subdivisions in a wide range of populations. 
Thin koniocellular layers ( $\mathrm{K}$ layers) are known to exist between each layer of the $\mathrm{M}$ and P subdivisions in the LGN (Guillery and Colonnier, 1970). Previous histological studies have indicated that the $\mathrm{K}$ layers have distinct anatomical properties compared with those in the $M$ and $P$ subdivisions (Hendry and Reid, 2000). Considering the location of $K$ layers, the MTV fractions in many LGN voxels are likely to be affected by partial volumes between the $\mathrm{K}$ layers and $\mathrm{M}$ or $\mathrm{P}$ subdivisions. Therefore, part of the variance in the structural measurements may be affected by the anatomical properties of the $\mathrm{K}$ layers.

Despite the limitations discussed above, this study provides an important step toward establishing a method of parcellating human LGN subdivisions and quantifying their tissue properties in living human brains. Once the limitation of acquisition time has been overcome, MTV-based in vivo human LGN measurements will create fruitful opportunities to investigate how the properties of the human LGN are related to brain functions and affected as a consequence of disease. For example, psychophysical studies have suggested that certain properties of the $M$ subdivision may be crucially related to reading performance (Chase and Jenner, 1993; Demb et al., 1998; Felmingham and Jakobson, 1995; Main et al., 2014; Stein, 2001). Also, psychophysical investigations of glaucoma patients have suggested that the $M$ subdivision may be damaged earlier than $P$ subdivision (Cello et al., 2000; Maddess et al., 1992). Extensions of this study will create a powerful tool for directly comparing LGN tissue properties with psychophysical performance to improve our understanding of how various properties of the LGN subdivisions are related to visual functions and disorders.

\section{Funding}

This work was supported by the Japan Society for the Promotion of Science (JSPS) KAKENHI (JP20J11101 to H.O., JP17H04684 to H.T.). The funders had no role in the study design, data collection and interpretation, or the decision to submit the work for publication.

\section{Acknowledgments}

We thank Yusuke Sakai for supporting the acquisition and analysis of the data.

\section{Competing interests}

The authors declare no competing interests. 


\section{References}

Amunts K, Lepage C, Borgeat L, Mohlberg H, Dickscheid T, Rousseau M-É, Bludau S, Bazin P-L, Lewis LB, Oros-Peusquens A-M, Shah NJ, Lippert T, Zilles K, Evans AC. 2013. BigBrain: an ultrahigh-resolution 3D human brain model. Science 340:14721475.

Andrews TJ, Halpern SD, Purves D. 1997. Correlated size variations in human visual cortex, lateral geniculate nucleus, and optic tract. Journal of Neuroscience 17:28592868.

Arshad M, Stanley JA, Raz N. 2017. Test-retest reliability and concurrent validity of in vivo myelin content indices: Myelin water fraction and calibrated T1 w/T2 w image ratio. Human Brain Mapping 38:1780-1790.

Ban H, Yamamoto H. 2013. A non-device-specific approach to display characterization based on linear, nonlinear, and hybrid search algorithms. J Vis 13(6):20.

Barral JK, Gudmundson E, Stikov N, Etezadi-Amoli M, Stoica P, Nishimura DG. 2010. A robust methodology for in vivo T1 mapping. Magn Reson Med 64:1057-1067.

Berman S, West KL, Does MD, Yeatman JD, Mezer AA. 2018. Evaluating g-ratio weighted changes in the corpus callosum as a function of age and sex. Neuroimage 182:304-313.

Boynton GM, Engel SA, Glover GH, Heeger DJ. 1996. Linear systems analysis of functional magnetic resonance imaging in human V1. J Neurosci 16:4207-4221.

Caan MWA, Bazin P-L, Marques JP, de Hollander G, Dumoulin SO, van der Zwaag W. 2019. MP2RAGEME: $\mathrm{T}_{1}, \mathrm{~T}_{2}{ }^{*}$, and QSM mapping in one sequence at 7 tesla. Hum Brain Mapp 40:1786-1798.

Cello KE, Nelson-Quigg JM, Johnson CA. 2000. Frequency doubling technology perimetry for detection of glaucomatous visual field loss. American Journal of Ophthalmology 129:314-322.

Cercignani M, Dowell NG, Tofts PS. 2018. Quantitative MRI of the Brain: Principles of Physical Measurement, Second edition. CRC Press.

Chase C, Jenner AR. 1993. Magnocellular visual deficits affect temporal processing of dyslexics. Ann N Y Acad Sci 682:326-329.

Csernansky JG, Schindler MK, Splinter NR, Wang L, Gado M, Selemon LD, RastogiCruz D, Posener JA, Thompson PA, Miller MI. 2004. Abnormalities of thalamic volume and shape in schizophrenia. Am J Psychiatry 161:896-902.

Demb JB, Boynton GM, Best M, Heeger DJ. 1998. Psychophysical evidence for a magnocellular pathway deficit in dyslexia. Vision Res 38:1555-1559. 
Denison RN, Vu AT, Yacoub E, Feinberg DA, Silver MA. 2014. Functional mapping of the magnocellular and parvocellular subdivisions of human LGN. Neuroimage 102 Pt 2:358-369.

Derrington AM, Lennie P. 1984. Spatial and temporal contrast sensitivities of neurones in lateral geniculate nucleus of macaque. J Physiol 357:219-240.

Duval T, Le Vy S, Stikov N, Campbell J, Mezer A, Witzel T, Keil B, Smith V, Wald LL, Klawiter E, Cohen-Adad J. 2017. g-Ratio weighted imaging of the human spinal cord in vivo. Neuroimage 145:11-23.

Felmingham KL, Jakobson LS. 1995. Visual and visuomotor performance in dyslexic children. Exp Brain Res 106:467-474.

Filo S, Shtangel O, Salamon N, Kol A, Weisinger B, Shifman S, Mezer AA. 2019. Disentangling molecular alterations from water-content changes in the aging human brain using quantitative MRI. Nature Communications 10:3403.

Fonov V, Evans AC, Botteron K, Almli CR, McKinstry RC, Collins DL, Brain Development Cooperative Group. 2011. Unbiased average age-appropriate atlases for pediatric studies. Neuroimage 54:313-327.

Fonov VS, Evans AC, McKinstry RC, Almli CR, Collins DL. 2009. Unbiased nonlinear average age-appropriate brain templates from birth to adulthood. Neurolmage 47, Supplement 1:S102.

Forstmann BU, de Hollander G, van Maanen L, Alkemade A, Keuken MC. 2016. Towards a mechanistic understanding of the human subcortex. Nat Rev Neurosci 18:57.

Giraldo-Chica M, Hegarty JP 2nd, Schneider KA. 2015. Morphological differences in the lateral geniculate nucleus associated with dyslexia. Neuroimage Clin 7:830-836.

Giraldo-Chica M, Schneider KA. 2018. Hemispheric asymmetries in the orientation and location of the lateral geniculate nucleus in dyslexia. Dyslexia 24:197-203.

Glasser MF, Coalson TS, Robinson EC, Hacker CD, Harwell J, Yacoub E, Ugurbil K, Andersson J, Beckmann CF, Jenkinson M, Smith SM, Van Essen DC. 2016. A multimodal parcellation of human cerebral cortex. Nature 536:171-178.

Glasser MF, Sotiropoulos SN, Wilson JA, Coalson TS, Fischl B, Andersson JL, Xu J, Jbabdi S, Webster M, Polimeni JR, Van Essen DC, Jenkinson M, Consortium, W. U-Minn HCP. 2013. The minimal preprocessing pipelines for the Human Connectome Project. Neuroimage 80:105-124.

Glasser MF, Van Essen DC. 2011. Mapping human cortical areas in vivo based on myelin content as revealed by T1- and T2-weighted MRI. J Neurosci 31:11597-11616.

Guillery RW, Colonnier M. 1970. Synaptic patterns in the dorsal lateral geniculate nucleus of the monkey. Z Zellforsch Mikrosk Anat 103:90-108. 
Hagiwara A, Hori M, Kamagata K, Warntjes M, Matsuyoshi D, Nakazawa M, Ueda R, Andica C, Koshino S, Maekawa T, Irie R, Takamura T, Kumamaru KK, Abe O, Aoki S. 2018. Myelin measurement: comparison between simultaneous tissue relaxometry, magnetization transfer saturation index, and $T_{1} w / T_{2} w$ ratio methods. Sci Rep 8:10554.

Hassler R. 1966. Comparative anatomy of the central visual systems in day- and nightactive primates. Evolution of the Forebrain. In: Hassler R., Stephan H. (eds) Evolution of the Forebrain. Springer, Boston, MA

Haynes J-D, Deichmann R, Rees G. 2005. Eye-specific effects of binocular rivalry in the human lateral geniculate nucleus. Nature 438:496-499.

Hendry SHC, Reid RC. 2000. The koniocellular pathway in primate vision. Annual Review of Neuroscience 23:127-153.

Hickey TL, Guillery RW. 1979. Variability of laminar patterns in the human lateral geniculate nucleus. J Comp Neurol 183:221-246.

Huber L, Ivanov D, Handwerker DA, Marrett S, Guidi M, Uludağ K, Bandettini PA, Poser BA. 2018. Techniques for blood volume fMRI with VASO: From low-resolution mapping towards sub-millimeter layer-dependent applications. Neuroimage 164:131-143.

Huber L, Ivanov D, Krieger SN, Streicher MN, Mildner T, Poser BA, Möller HE, Turner R. 2014. Slab-selective, BOLD-corrected VASO at 7 Tesla provides measures of cerebral blood volume reactivity with high signal-to-noise ratio. Magn Reson Med 72:137-148.

Hughes SW, Lörincz M, Cope DW, Blethyn KL, Kékesi KA, Parri HR, Juhász G, Crunelli V. 2004. Synchronized oscillations at alpha and theta frequencies in the lateral geniculate nucleus. Neuron 42:253-268.

Ives HE. 1912. XII. Studies in the photometry of lights of different colours. The London, Edinburgh, and Dublin Philosophical Magazine and Journal of Science 24:149-188. Jenkinson M, Bannister P, Brady M, Smith S. 2002. Improved optimization for the robust and accurate linear registration and motion correction of brain images. Neuroimage 17:825-841.

Joffe KM, Raymond JE, Chrichton A. 1997. Motion coherence perimetry in glaucoma and suspected glaucoma. Vision Res 37:955-964.

Kay K, Jamison KW, Vizioli L, Zhang R, Margalit E, Ugurbil K. 2019. A critical assessment of data quality and venous effects in sub-millimeter fMRI. Neuroimage 189:847-869.

Kay K, Jamison KW, Zhang R-Y, Uğurbil K. 2020. A temporal decomposition method for identifying venous effects in task-based fMRI. Nat Methods 17:1033-1039. 
Keuken MC, Bazin P-L, Backhouse K, Beekhuizen S, Himmer L, Kandola A, Lafeber JJ, Prochazkova L, Trutti A, Schäfer A, Turner R, Forstmann BU. 2017. Effects of aging on $\mathrm{T}_{1}, \mathrm{~T}_{2}{ }^{*}$, and $\mathrm{QSM} \mathrm{MRI}$ values in the subcortex. Brain Structure and Function 222:2487-2505.

Ling S, Pratte MS, Tong F. 2015. Attention alters orientation processing in the human lateral geniculate nucleus. Nature Neuroscience 18:496-498.

Liu Z, de Zwart JA, Yao B, van Gelderen P, Kuo L-W, Duyn JH. 2012. Finding thalamic BOLD correlates to posterior alpha EEG. Neuroimage 63:1060-1069.

Lutti A, Dick F, Sereno MI, Weiskopf N. 2014. Using high-resolution quantitative mapping of R1 as an index of cortical myelination. Neuroimage 93 Pt 2:176-188.

Maddess TL, Henry GH, Others. 1992. Performance of nonlinear visual units in ocular hypertension and glaucoma. Clin. Vision Sci. 7:371-383.

Ma D, Gulani V, Seiberlich N, Liu K, Sunshine JL, Duerk JL, Griswold MA. 2013. Magnetic resonance fingerprinting. Nature 495:187-192.

Main KL, Pestilli F, Mezer A, Yeatman J, Martin R, Phipps S, Wandell B. 2014. Speed discrimination predicts word but not pseudo-word reading rate in adults and children. Brain Lang 138:27-37.

Marques JP, Kober T, Krueger G, van der Zwaag W, Van de Moortele P-F, Gruetter R. 2010. MP2RAGE, a self bias-field corrected sequence for improved segmentation and T1-mapping at high field. Neuroimage 49:1271-1281.

Mcketton L, Kelly KR, Schneider KA. 2014. Abnormal lateral geniculate nucleus and optic chiasm in human albinism. Journal of Comparative Neurology 522:2680-2687.

Mezer A, Rokem A, Berman S, Hastie T, Wandell BA. 2016. Evaluating quantitative proton-density-mapping methods. Hum Brain Mapp 37:3623-3635.

Mezer A, Yeatman JD, Stikov N, Kay KN, Cho NJ, Dougherty RF, Perry ML, Parvizi J, Hua le H, Butts-Pauly K, Wandell BA. 2013. Quantifying the local tissue volume and composition in individual brains with magnetic resonance imaging. Nat Med 19:1667-1672.

Minami S, Amano K. 2017. Illusory jitter perceived at the frequency of alpha oscillations. Curr Biol 27:2344-2351.e4.

Minami S, Oishi H, Takemura H, Amano K. 2020. Inter-individual differences in occipital alpha oscillations correlate with white matter tissue properties of the optic radiation. eNeuro 7:ENEURO.0224-19.2020.

Moeller S, Yacoub E, Olman CA, Auerbach E, Strupp J, Harel N, Uğurbil K. 2010. Multiband multislice GE-EPI at 7 tesla, with 16 -fold acceleration using partial parallel 
imaging with application to high spatial and temporal whole-brain fMRI. Magn Reson Med 63:1144-1153.

Müller-Axt C, Eichner C, Rusch H, Kauffmann L, Bazin P-L, Anwander A, Morawski M, von Kriegstein K. 2020. Mapping the human visual thalamus and its cytoarchitectonic subdivisions using quantitative MRI. bioRxiv. doi:10.1101/2020.08.14.250779

Nassi JJ, Callaway EM. 2009. Parallel processing strategies of the primate visual system. Nat Rev Neurosci 10:360-372.

O'Connor DH, Fukui MM, Pinsk MA, Kastner S. 2002. Attention modulates responses in the human lateral geniculate nucleus. Nat Neurosci 5:1203-1209.

Oishi H, Takemura H, Aoki SC, Fujita I, Amano K. 2018. Microstructural properties of the vertical occipital fasciculus explain the variability in human stereoacuity. Proc Natl Acad Sci U S A 115:12289-12294.

Pistorio AL, Hendry SH, Wang X. 2006. A modified technique for high-resolution staining of myelin. J Neurosci Methods 153:135-146.

Schiller PH, Logothetis NK, Charles ER. 1990. Functions of the colour-opponent and broad-band channels of the visual system. Nature 343:68-70.

Schneider KA, Kastner S. 2009. Effects of sustained spatial attention in the human lateral geniculate nucleus and superior colliculus. J Neurosci 29:1784-1795.

Selemon LD, Begovic A. 2007. Stereologic analysis of the lateral geniculate nucleus of the thalamus in normal and schizophrenic subjects. Psychiatry Res 151:1-10.

Sereno MI, Lutti A, Weiskopf N, Dick F. 2013. Mapping the human cortical surface by combining quantitative $T_{1}$ with retinotopy. Cereb Cortex 23:2261-2268.

Shams Z, Norris DG, Marques JP. 2019. A comparison of in vivo MRI based cortical myelin mapping using T1w/T2w and R1 mapping at 3T. PLoS One 14:e0218089.

Shtangel O, Mezer AA. 2020. A phantom system for assessing the effects of membrane lipids on water proton relaxation. NMR Biomed 33:e4209.

Stein J. 2001. The magnocellular theory of developmental dyslexia. Dyslexia 7:12-36.

Stüber C, Morawski M, Schafer A, Labadie C, Wahnert M, Leuze C, Streicher M, Barapatre N, Reimann K, Geyer S, Spemann D, Turner R. 2014. Myelin and iron concentration in the human brain: a quantitative study of MRI contrast. Neuroimage 93 Pt 1:95-106.

Takemura H, Ogawa S, Mezer AA, Horiguchi H, Miyazaki A, Matsumoto K, Shikishima K, Nakano T, Masuda Y. 2019. Diffusivity and quantitative T1 profile of human visual white matter tracts after retinal ganglion cell damage. Neuroimage Clin 23:101826. 
Uddin MN, Figley TD, Marrie RA, Figley CR, CCOMS Study Group. 2018. Can $T_{1} w / T_{2} w$ ratio be used as a myelin-specific measure in subcortical structures? Comparisons between FSE-based $T_{1} w / T_{2} w$ ratios, GRASE-based $T_{1} w / T_{2} w$ ratios and multi-echo GRASE-based myelin water fractions. NMR Biomed 31. doi:10.1002/nbm.3868

Uludağ K, Blinder P. 2018. Linking brain vascular physiology to hemodynamic response in ultra-high field MRI. Neuroimage 168:279-295.

Usrey WM, Reid RC. 2000. Visual physiology of the lateral geniculate nucleus in two species of new world monkey: Saimiri sciureus and Aotus trivirgatis. J Physiol $\mathbf{5 2 3}$ Pt 3:755-769.

Viviano JD, Schneider KA. 2015. Interhemispheric interactions of the human thalamic reticular nucleus. J Neurosci 35:2026-2032.

Warntjes JBM, Leinhard OD, West J, Lundberg P. 2008. Rapid magnetic resonance quantification on the brain: optimization for clinical usage. Magn Reson Med 60:320329.

Weiskopf N, Mohammadi S, Lutti A, Callaghan MF. 2015. Advances in MRI-based computational neuroanatomy: from morphometry to in-vivo histology. Curr Opin Neurol 28:313-322.

Williams RW, Rakic P. 1988. Elimination of neurons from the rhesus monkey's lateral geniculate nucleus during development. J Comp Neurol 272:424-436.

Yeshurun Y, Levy L. 2003. Transient spatial attention degrades temporal resolution. Psychol Sci 14:225-231.

Yücel YH, Zhang Q, Gupta N, Kaufman PL, Weinreb RN. 2000. Loss of neurons in magnocellular and parvocellular layers of the lateral geniculate nucleus in glaucoma. Arch Ophthalmol 118:378-384.

Yücel YH, Zhang Q, Weinreb RN, Kaufman PL, Gupta N. 2003. Effects of retinal ganglion cell loss on magno-, parvo-, koniocellular pathways in the lateral geniculate nucleus and visual cortex in glaucoma. Prog Retin Eye Res 22:465-481.

Yu Q, Zhang P, Quu J, Fang F. 2016. Perceptual Learning of Contrast Detection in the Human Lateral Geniculate Nucleus. Curr Biol 26:3176-3182.

Zhang P, Wen W, Sun X, He S. 2016. Selective reduction of fMRI responses to transient achromatic stimuli in the magnocellular layers of the LGN and the superficial layer of the SC of early glaucoma patients. Hum Brain Mapp 37:558-569.

Zhang P, Zhou H, Wen W, He S. 2015. Layer-specific response properties of the human lateral geniculate nucleus and superior colliculus. Neuroimage 111:159-166. 